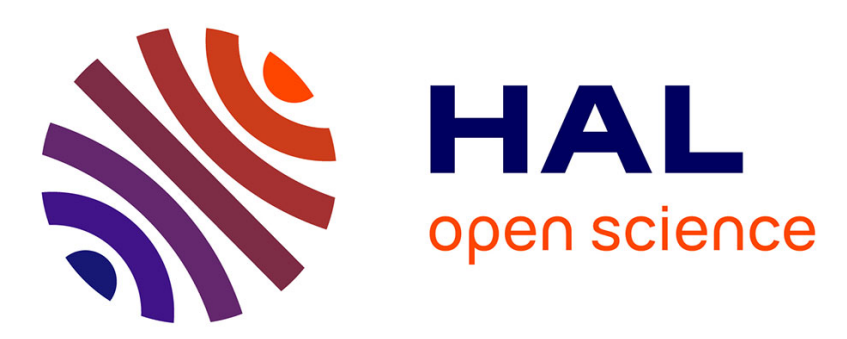

\title{
Statistics about collisions of ellipsoids under shear flow
}

\author{
Frédéric Gruy, Patrice Nortier
}

\section{To cite this version:}

Frédéric Gruy, Patrice Nortier. Statistics about collisions of ellipsoids under shear flow. Colloids and Surfaces A: Physicochemical and Engineering Aspects, 2018, 558, pp.250 à 262. 10.1016/j.colsurfa.2018.08.065 . hal-01872034

\section{HAL Id: hal-01872034 https://hal.science/hal-01872034}

Submitted on 7 Sep 2021

HAL is a multi-disciplinary open access archive for the deposit and dissemination of scientific research documents, whether they are published or not. The documents may come from teaching and research institutions in France or abroad, or from public or private research centers.
L'archive ouverte pluridisciplinaire $\mathbf{H A L}$, est destinée au dépôt et à la diffusion de documents scientifiques de niveau recherche, publiés ou non, émanant des établissements d'enseignement et de recherche français ou étrangers, des laboratoires publics ou privés. 


\title{
Statistics about Collisions of Ellipsoids under Shear Flow
}

\author{
Frédéric Gruy (*), Patrice Nortier \\ Mines Saint-Etienne, Univ Lyon, CNRS, UMR 5307 LGF, Centre SPIN, F - 42023 Saint-
}

Etienne France

* corresponding author

gruy@emse.fr

0033477420202

\begin{abstract}
:
Shear aggregation is an important phenomenon occurring during particle precipitation in a stirred chemical reactor. It concerns micro-particles that are often non-spherical or aggregated. Modelling of precipitation involves the selection of internal parameters describing the particle. Among them, morphological or geometrical descriptors are required. In this paper, the inertia or gyration tensor of primary particles and of their cluster is considered and evaluated for the modelling of aggregation. An equivalent ellipsoid is defined from the inertia tensor. In this framework, the collision event becomes the collision between two equivalent ellipsoids and leads to a larger ellipsoid. The inertia tensor and the semi-axis lengths of the equivalent ellipsoid are explicitly calculated in the case of shear aggregation in a three-dimensional space. Two ways for performing these calculations are presented. The statistics of the equivalent ellipsoids associated with the set of collision events undergone by two given ellipsoids have been computed. Statistics includes the probability density for geometrical parameters and the average value. Collision rate constant, i.e. aggregation kernel, is also calculated. Corresponding approximate expressions are presented and checked. Finally, equivalence
\end{abstract}


between the sphere cluster and the equivalent ellipsoid with respect to the aggregation act is checked and ascertained.

Keywords: aggregation, inertia tensor, probability density, equivalent ellipsoid, aggregation kernel

\section{Nomenclature (main notations)}

a translation vector for particle

$a, b, c \quad$ semi-axes of ellipsoid

$\mathrm{A}_{\mathrm{k}} \quad$ random numbers $(\mathrm{k}=1-4)$

$\mathbf{A}, \mathbf{A}_{\mathbf{E}}$ ellipsoid matrices

b $\quad b_{1}=-c_{1} \quad b_{2}=c_{2}$

c position vector of ellipsoid center

C center of mass

$C_{i}, C_{i, j}$ fitting parameters

$\mathbf{D}_{\mathbf{E}} \quad$ inertia tensor for ellipsoid

$\mathbf{D}_{\mathbf{I}} \quad$ diagonalized inertia tensor

$\overline{\mathbf{D}_{\mathbf{1}}}$ diagonalized inertia tensor after averaging

$\mathbf{D}_{\mathbf{q}} \quad$ tensor $(q=y, z ;$ Eq.23 $)$

f diagonal matrix (Eq.29)

F tensor (Eq.32)

$\mathbf{i}, \mathbf{j}, \mathbf{k}$ unit vectors

I inertia tensor of the particle 
$k, l \quad$ aspect ratii of ellipsoid

$k_{12} \quad$ contribution to $K_{12}$

$K_{12} \quad$ kernel of aggregation

$m \quad$ particle mass

MC Monte Carlo

$\mathrm{N}$ number of primary particle in the cluster

$N_{c} \quad$ number of effective collisions (MC)

$N_{r} \quad$ number of events (MC)

pdf probability density function

$P \quad$ point of tangency

$P(k, l), Q(k, l):$ intermediate variables (Eqs.28d-f)

p position vector on ellipsoid surface

$R \quad$ sphere radius

$R_{g} \quad$ gyration radius

r position vector

$\mathbf{R}$ rotation matrix

$S_{p} \quad$ projected surface area

u normal vector

$\mathbf{U} \quad$ unit tensor

$x, y, z: \quad$ space coordinates

$X \quad$ size parameter (Eq.28a)

Greek letters

$\dot{\gamma} \quad$ shear rate

$\phi \quad=m_{\mathrm{i}} /\left(m_{1}+m_{2}\right)$ 
$\lambda \quad$ eigenvalue

$\rho \quad$ mass density

$\Psi=\left(\psi_{y}, \psi_{z}\right) \quad$ rotation angles for vectors

$\Theta=\left(\theta_{y}, \theta_{z}, \theta_{x}\right) \quad$ Euler angles for solids

$\sigma \quad$ relative standard deviation

$\sum\left(\Theta_{1}, \Theta_{2}\right) \quad$ collision area

\section{Superscript}

$\mathrm{T} \quad$ transpose

\section{Subscript}

approx approximation (Eqs. 28a-f)

CC sphere cluster - sphere cluster collision

EE ellipsoid - ellipsoid collision

$i \quad$ for scalar, vector, tensor (particle $i=1,2$ ) or for index of series

12 for scalar, vector, tensor (particle set, 1 and 2)

proj corresponds to the method developed in section 3

pv corresponds to the method developed in section 2

S relative to Smoluchowski

symbol

$<\quad$ average 


\section{Introduction}

Aggregation of particles in a suspension is due to the binary collisions between two particles. Resulting aggregate or cluster is a branched and porous object consisting of primary particles, usually considered as identical. The morphology of the cluster is usually described in terms of the fractal theory: it is defined by the radius of its primary particle, the number of primary particles and the fractal dimension [1]. The last quantity is a real number within the 1-3 range. Three basic collision mechanisms have been identified: Brownian for nanoparticles and colloids, shear and differential settling for micro-particles. The fractal dimension value depends on the mechanism details [2]. Even if the fractal hypothesis was successful for describing the dynamics of aggregation, the fractal dimension is not the only relevant morphological parameter. Indeed, the shape of the colliding bodies or more precisely the shape of the convex hull is also a major factor for the encounter efficiency [3].

Torres et al. [4] have, for instance, performed simulations of 3-D shear aggregation. They found a fractal dimension value equal to 1.83 and they observed a non-spherical shape of the aggregate. Moreover they have calculated the inertia tensor (normalized by the square of the gyration radius) for clusters with a number $N$ of primary particles smaller than 4100 . They have observed that the semi-axes of the equivalent ellipsoid were in the ratio 1/1.6/3.1 independently of the origin of the particle collision (Brownian motion, shear flow, elongation flow). Applying the coupling between Stokesian Dynamics and Discrete Element Method to sphere-like particle aggregation under shear flow, Frungieri and Vanni [5] proved the importance of the aspect ratio of the aggregate. The aspect ratio has been calculated from the inertia ellipsoid of the sphere cluster. This fact has been experimentally observed for particle cluster larger than one micrometre [6]. Independently, 
Gruy laid the foundations of a new modelling method for the aggregation based on the equivalent ellipsoid of colliding aggregates [7]. This has been illustrated in the simplest case: the 2D-aggregation of equivalent ellipses of 2D-clusters under shear flow. According to all of the aforementioned researchers the crucial point is the collision of two ellipsoids taking into account the stochastic behaviour of the aggregation in suspensions containing a very large number of particles. Herein, ellipsoid in this sense means convex ellipsoidal particle or equivalent ellipsoid for a cluster of spheres. This paper is dedicated to a fine description of the collision of two ellipsoids and the resulting equivalent ellipsoid. It appears that the application of the new approach [7] to 3D-shear aggregation leads to tricky calculations requiring some careful validation. Replacing sphere by ellipsoid for representing any object drastically increases the difficulty level when trying to model the physical phenomena of interest. This has been already underlined by, for instance, [8]. Therefore two methods for performing collision of ellipsoids under shear flow have been proposed and compared: quasi-analytical calculations and Monte-Carlo simulations. Our goal is to determine the collision dynamics and the parameters of the resulting equivalent ellipsoid. As a result, some empirical expressions aiming at accelerating the simulations of particle population dynamics [9] will be proposed.

The Section $2(\S 2)$ presents the theoretical background on the collision of two ellipsoids under shear flow. This will be hereinafter referred to as quasi-analytical method. The principles of Monte-Carlo approach are explained in the section $3(\S 3)$. This will be hereinafter referred to as Monte Carlo method. The section 4 is dedicated to the results concerning the collision of ellipsoids and the statistics relative to the resulting body. The kinetic and morphological aspects for the collision between clusters of primary particles and collision of their equivalent ellipsoids are compared there. The conclusion is presented in the Section 5. 


\section{Theory on the collision of two ellipsoids under shear flow}

In this section we establish the fundamentals about the collision between two ellipsoids under shear flow and the characteristics of the resulting object. This will lead to analytical expressions containing integrals that must be later numerically evaluated.

\subsection{Notations}

In the following text, lower case is used to denote scalar quantities, while bold lower case characters correspond to vectors and bold uppercase characters to matrices or secondorder tensors.

Let us consider (Figure 1) the orthonormal system with $z$ aligned in the flow direction (unit vector $\mathbf{k}$ ), $y$ perpendicular to the flow and parallel to the velocity gradient (unit vector $\mathbf{j}$ ), $x$ to complete the orthonormal system (unit vector $\mathbf{i}$ ). The rotation of a unit vector will be defined by two angles $\Psi=\left(\psi_{y}, \psi_{z^{\prime}}\right): \psi_{y}$ denoting the rotation angle around $y, \quad x \rightarrow x^{\prime} y=y^{\prime} z \rightarrow z^{\prime} \quad, \quad \psi_{z^{\prime}} \quad$ denoting the rotation angle around $z^{\prime}$, $x^{\prime} \rightarrow x^{\prime \prime} y^{\prime} \rightarrow y^{\prime \prime} z^{\prime \prime}=z^{\prime}$. The corresponding rotation matrices are:

$\mathbf{R}_{\Psi}=\mathbf{R}_{\mathbf{z}^{\prime}}\left(\psi_{z}\right) \mathbf{R}_{\mathbf{y}}\left(\psi_{y}\right)$ or $\mathbf{R}_{\mathbf{\Psi}}=\mathbf{R}_{\mathbf{y}}\left(\psi_{y}\right) \mathbf{R}_{\mathbf{z}}\left(\psi_{z}\right)$ with $\Psi=\left(\psi_{y}, \psi_{z}\right)$

In the same vein, the rotation of an ellipsoid can be defined from the Tait-Bryan angle triplet [10] $\Theta=\left(\theta_{y}, \theta_{z}, \theta_{x}\right): \theta_{y}$ denoting the rotation angle around $y$, $x \rightarrow x^{\prime} y=y^{\prime} z \rightarrow z^{\prime}, \theta_{z^{\prime}}$ denoting the rotation angle around $z^{\prime}, x^{\prime} \rightarrow x^{\prime \prime} y^{\prime} \rightarrow y^{\prime \prime} z^{\prime \prime}=z^{\prime}$, $\theta_{x^{\prime \prime}}$ being the rotation angle around $x^{\prime \prime}, x^{\prime \prime \prime}=x^{\prime \prime} y^{\prime \prime} \rightarrow y^{\prime \prime} z^{\prime \prime} \rightarrow z^{\prime \prime \prime}$

Then,

$\mathbf{R}_{\mathbf{\Theta}}=\mathbf{R}_{\mathbf{x}^{\prime \prime}}\left(\theta_{x}\right) \mathbf{R}_{\mathbf{z}^{\prime}}\left(\theta_{z}\right) \mathbf{R}_{\mathbf{y}}\left(\theta_{y}\right)$ or $\mathbf{R}_{\boldsymbol{\Theta}}=\mathbf{R}_{\mathbf{y}}\left(\theta_{y}\right) \mathbf{R}_{\mathbf{z}}\left(\theta_{z}\right) \mathbf{R}_{\mathbf{x}}\left(\theta_{x}\right)$.

It may be underlined that other angle triplets can be defined and used instead of TaitBryan angles [11]. 
An ellipsoid is characterized by the three semi-axes $(a \geq b \geq c)$; its position is defined by the coordinates of the center, the generalized Tait-Bryan angles $\Theta$ to rotate it from the reference position $a \square x, b \square y, c \square z$ to its current position $a \square x^{\prime \prime \prime}, b \square y^{\prime \prime \prime}, c \square z$ "'.

The ellipsoid itself is described by the position vector $\mathbf{c}$ of its center $C$ and a matrix containing the semi-axes:

$\mathbf{A}_{\mathbf{E}}=\left[\begin{array}{ccc}a^{-2} & 0 & 0 \\ 0 & b^{-2} & 0 \\ 0 & 0 & c^{-2}\end{array}\right]$

A point on the ellipsoid surface satisfies the vector equation:

$$
(\mathbf{r}-\mathbf{c})^{\mathrm{T}} \mathrm{A}(\mathbf{r}-\mathbf{c})=1
$$

With

$$
\mathbf{A}=\mathbf{R}_{\boldsymbol{\Theta}} \mathbf{A}_{\mathbf{E}} \mathbf{R}_{\boldsymbol{\Theta}}^{\mathbf{T}}
$$

\subsection{Contact between two ellipsoids}

Let us consider two ellipsoids E1 and E2. The center $C_{l}$ of E1 matches with the origin of the orthonormal system. E1 is rotated by the angle $\Theta_{1}$. The position of the E2 center is called $C_{2}$. E2 is rotated by the angle $\Theta_{2}$. Let $P$ be the point of tangency and $\mathbf{u}$ the normal vector to the tangent plane: $\mathbf{u}$ is characterized by $\Psi$. Then,

$$
\mathbf{u}=\mathbf{R}_{\Psi} \mathbf{i}
$$

$P$ complies with the two conditions for each ellipsoid $(\mathrm{i}=1,2)$ :

i) $\quad P$ is located on $\mathrm{Ei}$ :

$$
\left(\mathbf{p}-\mathbf{c}_{\mathbf{i}}\right)^{\mathbf{T}} \mathbf{A}_{\mathbf{i}}\left(\mathbf{p}-\mathbf{c}_{\mathbf{i}}\right)=1
$$

With 
$\mathbf{A}_{\mathrm{i}}=\mathbf{R}_{\Theta \mathrm{i}} \mathbf{A}_{\mathrm{E}, \mathrm{i}} \mathbf{R}_{\Theta, \mathrm{i}}^{\mathrm{T}}$

ii) The surface of the ellipsoid is normal to $\mathbf{u}$ in $P$ :

$$
\nabla\left[\left(\mathbf{p}-\mathbf{c}_{\mathbf{i}}\right)^{\mathbf{T}} \mathbf{A}_{\mathbf{i}}\left(\mathbf{p}-\mathbf{c}_{\mathbf{i}}\right)\right]=\lambda_{i} \mathbf{u}
$$

Where $\lambda_{i}$ is a scalar to be determined.

As $\mathbf{A}_{\mathbf{i}}$ is a symmetrical matrix:

$$
\nabla\left[\left(\mathbf{p}-\mathbf{c}_{\mathbf{i}}\right)^{\mathbf{T}} \mathbf{A}_{\mathbf{i}}\left(\mathbf{p}-\mathbf{c}_{\mathbf{i}}\right)\right]=2 \mathbf{A}_{\mathbf{i}}\left(\mathbf{p}-\mathbf{c}_{\mathbf{i}}\right)
$$

Then,

$$
\mathbf{p}-\mathbf{c}_{\mathbf{i}}=\left(\lambda_{i} / 2\right) \mathbf{A}_{\mathbf{i}}^{-1} \mathbf{u}
$$

By using (5) one deduces:

$$
\lambda_{i}=2 \varepsilon_{i} / \sqrt{\mathbf{u}^{\mathbf{T}} \mathbf{A}_{\mathbf{i}}^{-1} \mathbf{u}}
$$

With $\varepsilon_{i}= \pm 1$

As $\mathbf{u}$ is the outward-pointing normal to E1, $\varepsilon_{1}=+1$ and $\varepsilon_{2}=-1$

Finally, one deduces that:

$$
\mathbf{c}_{12}=\mathbf{c}_{2}-\mathbf{c}_{1}=\mathbf{b}_{1}+\mathbf{b}_{2}=\frac{\mathbf{A}_{1}^{-1} \mathbf{u}}{\sqrt{\mathbf{u}^{\mathrm{T}} \mathbf{A}_{1}^{-1} \mathbf{u}}}+\frac{\mathbf{A}_{2}^{-1} \mathbf{u}}{\sqrt{\mathbf{u}^{\mathrm{T}} \mathbf{A}_{2}^{-1} \mathbf{u}}}=\frac{\mathbf{A}_{1}^{-1} \mathbf{R}_{\Psi} \mathbf{i}}{\sqrt{\mathbf{i}^{\mathrm{T}} \mathbf{R}_{\Psi}^{\mathrm{T}} \mathbf{A}_{1}^{-1} \mathbf{R}_{\Psi} \mathbf{i}}}+\frac{\mathbf{A}_{2}^{-1} \mathbf{R}_{\Psi} \mathbf{i}}{\sqrt{\mathbf{i}^{\mathrm{T}} \mathbf{R}_{\Psi}^{\mathrm{T}} \mathbf{A}_{2}^{-1} \mathbf{R}_{\Psi} \mathbf{i}}}
$$

\subsection{Calculation of the parameters of the two-ellipsoids set}

Let us consider two objects with the inertia tensors $\mathbf{I}_{\mathbf{i}}$ and mass $m_{i}(i=1,2)$, each one evaluated about its own center of mass $C_{i}$.

The inertia tensor of an ellipsoid, located at the reference position ( $a \square x, b \square y, c \square z$ ), has the diagonal form: 
$\mathbf{D}_{\mathbf{E}}=\frac{m}{5}\left[\begin{array}{ccc}b^{2}+c^{2} & 0 & 0 \\ 0 & a^{2}+c^{2} & 0 \\ 0 & 0 & a^{2}+b^{2}\end{array}\right]$

Or

$\mathbf{D}_{\mathbf{E}}=\frac{m}{5}\left(\operatorname{Tr}\left(\mathbf{A}_{\mathbf{E}}^{-1}\right) \mathbf{U}-\mathbf{A}_{\mathbf{E}}^{-1}\right)$

$\operatorname{Tr}()$ is the trace of the matrix. $\mathbf{U}$ is the unit matrix.

With the notations (sub-section 2.1), the inertia tensor of each rotated ellipsoid $(i=1,2)$ obeys the relation:

$\mathbf{I}_{\mathbf{i}}=\mathbf{R}_{\Theta i} \mathbf{D}_{\mathbf{E}, \mathbf{i}} \mathbf{R}_{\Theta, \mathrm{i}}^{\mathrm{T}}$

The inertia tensor evaluated at another point $P\left(\mathbf{a}_{\mathbf{i}}\right.$ is the line segment from $P$ to $\left.C_{i}\right)$ becomes [12]:

$\mathbf{I}_{\mathbf{i}}{ }^{\prime}=\mathbf{I}_{\mathbf{i}}+m_{i}\left(\mathbf{a}_{\mathbf{i}}^{2} \mathbf{U}-\mathbf{a}_{\mathbf{i}} \mathbf{a}_{\mathbf{i}}\right)$

As a consequence, the inertia tensor of the object resulting from the collision of two smaller ellipsoids with the centers of mass separated by $\mathbf{c}_{\mathbf{1 2}}$ obeys the equation:

$\mathbf{I}_{\mathbf{1 2}}=\mathbf{I}_{1}+\mathbf{I}_{\mathbf{2}}+m_{1} m_{2} /\left(m_{1}+m_{2}\right)\left(\mathbf{c}_{\mathbf{1 2}}^{\mathbf{2}} \mathbf{U}-\mathbf{c}_{\mathbf{1 2}} \mathbf{c}_{\mathbf{1 2}}\right)$

The principal moments of inertia of the resulting object are obtained from the diagonalization of the tensor $\mathbf{I}_{\mathbf{1 2}}$; the corresponding diagonalized tensor is denoted by $\mathbf{D}_{\mathbf{I}, \mathbf{1 2}}$. If the eigenvalues of $\mathbf{I}_{12}$ are denoted by $\lambda_{1}, \lambda_{2}, \lambda_{3}\left(\lambda_{1}>\lambda_{2}>\lambda_{3}\right)$, the equivalent ellipsoid with demi-axes $a_{12}, b_{12}, c_{12}\left(a_{12}>b_{12}>c_{12}\right)$ is such as :

$$
\begin{aligned}
& \left(m_{1}+m_{2}\right) c_{12}^{2}=\frac{5}{2}\left(\lambda_{2}+\lambda_{3}-\lambda_{1}\right) \\
& \left(m_{1}+m_{2}\right) b_{12}^{2}=\frac{5}{2}\left(\lambda_{1}+\lambda_{3}-\lambda_{2}\right) \\
& \left(m_{1}+m_{2}\right) a_{12}^{2}=\frac{5}{2}\left(\lambda_{1}+\lambda_{2}-\lambda_{3}\right)
\end{aligned}
$$


In sum, one replaces the two colliding, i.e attached, ellipsoids by the equivalent ellipsoid having the same principal moments of inertia; their values depend on the contact point, i.e. $\mathbf{c}_{12}$, and on the orientations of the two ellipsoids.

\subsection{Aggregation kernel}

The assumptions of the collision model are the same as in our previous papers $[7,13]$ :

- The ellipsoids have no preferred orientation in a shear flow just before collision and, as a rough approximation, the orientation angle distribution of a single ellipsoid is uniform. This assumption has been discussed in [7].

- The hydrodynamic resistance [14-15] is neglected.

$K_{12}$ will be the notation for the collision kernel, i.e. the collision rate constant. The shear rate is denoted by $\dot{\gamma}$.

One considers a fixed orientation $\left(\Theta_{1}, \Theta_{2}\right)$ for each ellipsoid. $d k_{12}$ is then the contribution of an infinitesimal interception or collision area to the kernel. One performs the sum $k_{12}$ of all the contributions over the whole collision area. This area, that depends on the orientations of the two particles, is denoted by $\sum\left(\Theta_{1}, \Theta_{2}\right)$. As a consequence, the kernel obeys the relation:

$$
\begin{aligned}
& K_{12}=\left\langle k_{12}\right\rangle_{\left(\Theta_{1}, \Theta_{2}\right)} \\
& k_{12}=\int_{\sum\left(\Theta_{1}, \Theta_{2}\right)} d k_{12}
\end{aligned}
$$

The average \langle\rangle$_{\left(\Theta_{1}, \Theta_{2}\right)}$ is performed over all the orientations of the two ellipsoids. More precisely,

$$
k_{12}=\int_{\sum\left(\Theta_{1}, \Theta_{2}\right)} d k_{12}=2 \dot{\gamma} \int_{\psi_{y}=0}^{\pi} \int_{\psi_{z}=-\pi / 2}^{\pi / 2} \sup \left\{0, c_{2, y}-c_{1, y}\right\} d S_{p}
$$


where $c_{2, y}-c_{1, y}$ is the component of $\mathbf{c}_{\mathbf{1 2}}$ along y-axis, $d S_{p}$ is the element of area swept by the orthogonal projection along $z$ of $C 2$ in the plane xOy. $d S_{p}$ obeys the equation:

$$
d S_{p}=\left|\left(\frac{\partial \mathbf{c}_{\mathbf{1 2}}}{\partial \psi_{y}} \wedge \frac{\partial \mathbf{c}_{\mathbf{1 2}}}{\partial \psi_{z}}\right) \bullet \mathbf{k} d \psi_{y} d \psi_{z}\right|
$$

or

$$
d S_{p}=\left|\sum_{l, m=1}^{2}\left(\frac{\partial \mathbf{b}_{\mathbf{1}}}{\partial \psi_{y}} \wedge \frac{\partial \mathbf{b}_{\mathbf{m}}}{\partial \psi_{z}}\right) \bullet \mathbf{k} d \psi_{y} d \psi_{z}\right|
$$

with

$$
\frac{\partial \mathbf{b}_{1}}{\partial \psi_{q}}=\frac{1}{2\left(\mathbf{u}^{\mathrm{T}} \mathbf{A}_{1}^{-1} \mathbf{u}\right)^{3 / 2}} \mathbf{A}_{1}^{-1} \mathbf{D}_{\mathbf{q}} \mathbf{A}_{1}^{-1} \mathbf{u}
$$

where the two matrices $\mathbf{D}_{\mathbf{y}}, \mathbf{D}_{\mathbf{z}}$ are functions of $\Psi$ and detailed in annex 1 .

\subsection{Average resulting ellipsoid}

In practice, aggregation involves a large number of particles; a sub-set of particles has the same morphological characteristics, but with different orientations. Accordingly, the description of a collision between two objects with different morphological parameters

needs the average over all the possible orientations of the two objects. Therefore $\mathbf{D}_{\mathbf{I}, 12}$ must be firstly calculated for each pair of orientations. Moreover, the relative position $\mathbf{c}_{\mathbf{1 2}}$ of the centers of mass at impact depends both on the orientations of the two objects and on the mechanism of collision: a $\mathbf{c}_{\mathbf{1 2}}$ probability density could be introduced. The figure 2 includes some possible events. In the case of shear aggregation, the probability of encounter is all the higher as the two particles are offset to the side in the shear flow. A frontal impact is unlikely. In fact, the corresponding probability density is proportional to $d k_{12}$. This quantity was preferred to $\mathbf{c}_{\mathbf{1 2}}$ probability density. 
Keeping in mind that the orientations of two colliding objects are assumed non-correlated, i.e. the hydrodynamic resistance between two particles moving towards each other is not taken into account, and that the orientation angle distribution of a particle in a fluid is assumed to be uniform, one may write:

$\overline{\mathbf{D}_{\mathbf{I}, \mathbf{1 2}}}=\left\langle\int_{\Sigma\left(\Theta_{1}, \Theta_{2}\right)} \mathbf{D}_{\mathbf{I}, \mathbf{1 2}} d k_{12}\right\rangle_{\left(\Theta_{1}, \Theta_{2}\right)} / K_{12}$

The mean quantities \langle\rangle$_{\Theta_{i}}$ are performed over all the orientations. The semi-axis lengths of the average equivalent ellipsoid are easily deduced from $\overline{\mathbf{D}_{\mathbf{I}, 12}}$ (Eqs.17 and 24). The software corresponding to section 2 will be provided upon request.

\section{Monte Carlo Simulations for two bodies colliding under shear flow}

The collision of two objects has been also simulated by a Monte Carlo method with $N_{r}$ runs, each one having the following steps:

One considers a cube with the edge $a$ at the center of which is located the Cartesian coordinate system. The $a$ value is taken equal to $4 \times \max \left(a_{1}, a_{2}\right)$.

i. The particle 1 is located at the center of the coordinate system. Its orientation given by the Tait-Bryan angles $\Theta_{1}$ (three random numbers) is randomly selected.

ii. The particle 2 , the orientation of which is randomly chosen too ( using three random numbers corresponding to the Tait-Bryan angles $\Theta_{2}$ ), is located inside the cube. Given four random numbers $A_{1}, A_{2}, A_{3}$ et $A_{4}$ within the $[0 ; 1]$ range, the coordinates of the center of the particle 2 are: 


$$
\begin{aligned}
& X_{2}=\left(2 A_{3}-1\right) a / 2 \\
& Y_{2}=\frac{2 A_{1}-1}{\left|2 A_{1}-1\right|} \sqrt{A_{2}} a / 2 \\
& Z_{2}=\left(2 A_{4}-1\right) a / 2
\end{aligned}
$$

The expression for the $y$-coordinate of the particle 2 satisfies the likelihood of relative position between particles 1 and 2 in a shear flow [16].

iii. The particle shapes are projected onto the $x O y$ plane perpendicularly to $O z$. The figures generated by the projection are ellipses. Any intersection between the projections of particles 1 and 2 is searched. The collision will be effective if the two projections overlap and if $Y_{2} Z_{2}<0$. The latter condition means that the particle 2 is catching up with the particle 1 or is being caught up by particle 1: $Y_{2}>0 Z_{2}<0$ or $Y_{2}<0 Z_{2}>0$. The considered particle pairs require to perform the calculation of the intersection between two ellipses. This leads to the search of zeros of a quartic polynomial, for which the procedure of Rees has been used [17].

iv. The search of the contact point is performed as follows: in case of effective collision (see step iii)) and assuming the particle 2 located far upstream from the particle 1 , one considers the intersection between a line parallel to $\mathbf{k}$ and the two ellipsoids. The line is randomly chosen while intersecting the intersection area of the two overlapping ellipses. The line successively intersects the particle 2 at points $\mathrm{P} 2, \mathrm{Q} 2$ and the particle 1 at points $\mathrm{P} 1, \mathrm{Q} 1$. The distance between Q2 and P1 is calculated. Then, one search among a line beam the specific line having the minimum distance between Q2 and P1. The coordinates of the contact point and the components of $\mathbf{c}_{\mathbf{1 2}}$ are easily deduced. Finally, the diagonalized inertia tensor $\mathbf{D}_{\mathbf{I}, \mathbf{1 2}}$ is calculated as in $\S 2.3$ (Eq.16).

Only the first three steps are required for calculating the collision rate constant. By repeating these steps $N_{r}$ times, one may express the collision rate constant as:

$$
K_{12}=\left(\sum_{i=1}^{N r} \delta_{i} / N_{r}\right)\left(a^{3} / 2\right)=\left(N_{c} / N_{r}\right)\left(a^{3} / 2\right)
$$

with $\dot{\gamma}=1$ 
In case of collision $\delta_{i}=1$, if not $\delta_{i}=0 . N_{C}$ is the number of effective collisions. $N_{r}$ is taken equal to $10^{6}$, but may be modified in order to obtain the required accuracy (see below).

On the other hand, in order to calculate the diagonalized inertia tensor, which defines the equivalent ellipsoid of the two-ellipsoids set, the fourth step is needed. Then, the diagonalized inertia tensor of the average equivalent ellipsoid will be obtained from these ones got from each MC run (Eq. 27):

$$
\overline{\mathbf{D}_{\mathbf{I}, \mathbf{1 2}}}=\sum_{i=1}^{N r}\left(\delta_{i} / N_{c}\right)\left[\begin{array}{ccc}
\lambda_{1, i} & 0 & 0 \\
0 & \lambda_{2, i} & 0 \\
0 & 0 & \lambda_{3, i}
\end{array}\right] \quad \text { with } \quad \lambda_{1, i}>\lambda_{2, i}>\lambda_{3, i}
$$

As mentioned in the introduction, a cluster of sphere-like primary particles can be roughly represented by its equivalent ellipsoid. In order to compare the dynamics of two colliding clusters and their equivalent ellipsoids, the collision of sphere clusters has been also studied by means of Monte Carlo simulations. The procedure for clusters uses the same steps i) and ii) as for ellipsoids. Step iii) considers the projection (onto xOy) of all spheres belonging to the two clusters. If a pair of spheres exists (one allocated to the cluster 1, the other one to the cluster 2) such as the distance between the centers of their projection is smaller than the sphere diameter, then collision may occur and $\delta_{i}=1$; if not, $\delta_{i}=0$. Several pairs of spheres can lead to a bonding (bonding pair). The step iv) corresponds to the search of the contact point. One considers the two orientated clusters with the centers of mass located at the origin; one calculates the inter-center distance between each bonding pair of spheres. We select the bonding pair with the largest distance. Therefore, the two original clusters will meet and make contact when the spheres of this specific pair hit each other.

The software corresponding to section 3 will be provided upon request. 


\section{Results}

We will examine successively the collision rate constant $K_{12}$ between two ellipsoids and the parameters of the equivalent ellipsoid of the resulting body.

\section{1 kernel for ellipsoids collision}

The two calculation methods ( $\$ 2$ and $\S 3)$ have been applied to a set of two colliding ellipsoids. Ellipsoids 1 and 2 are such as:

$$
\begin{aligned}
& a_{1}=1 \quad b_{1} / a_{1} \in S \quad c_{1} / a_{1} \in S \quad c_{1} \leq b_{1} \\
& a_{2} \in\{1,2,5,10,15,20\} \quad b_{2} / a_{2} \in S \quad c_{2} / a_{2} \in S \quad c_{2} \leq b_{2}
\end{aligned}
$$

With $S=\{0.01 ; 0.1 ; 0.2 ; 0.5 ; 0.9\}$

The lengths are made dimensionless by the largest semi-axis of the smaller ellipsoid $\left(a_{1}=1\right)$.

Accordingly, we have considered $N=1350$ ellipsoid pairs, each ellipsoid may be rounded, elongated or flat in shape. $K_{12}$ values range between 0.7 and $10^{4}$.

Figure 3 compares the $K_{12}$ values based on Eqs. ( $\left.\$ 2\right)$ and denoted by $K_{12} p v$ with the $K_{12}$ values based on MC simulations ( $\left.\$ 3\right)$ and denoted by $K_{12}$ proj. It can be observed that the agreement between the two methods is very good. The relative standard deviation defined as $\sigma=\left(\sum_{i=1}^{N}\left(K_{12 p r o j}^{i} / K_{12 p v}^{i}-1\right)^{2} / N\right)^{1 / 2}$ is equal to 0.035 .

In a previous paper [13] we established an empirical expression for the collision rate constant valid for particles having a simple shape: bodies with high symmetry as sphere, spheroids, needles, discs. The collision rate constant may be expressed as:

$K_{12}=\left[4 / 3 \dot{\gamma}\left(X_{1}+X_{2}\right)^{3}\right] K_{12, N}\left(X_{1}, X_{2}\right)$

with

$$
X_{i}=\sqrt{\left(a_{i}^{2}+b_{i}^{2}+c_{i}^{2}\right) / 3}
$$




$$
\begin{aligned}
& h=X_{2} / X_{1} \\
& K_{12, N}(h)=\frac{1}{1+h^{n}}\left(P\left(k_{1}, l_{1}\right)+\left[\left(h^{m}+h^{-m}\right) / 2\right]^{-p} Q\left(k_{1}, l_{1}\right)\right) \\
& +\frac{1}{1+h^{-n}}\left(P\left(k_{2}, l_{2}\right)+\left[\left(h^{m}+h^{-m}\right) / 2\right]^{-p} Q\left(k_{2}, l_{2}\right)\right) \\
& P(k, l)=P_{0}+P_{1} \frac{k+l}{2}+P_{2}\left(\frac{k+l}{2}\right)^{3}+P_{3}(k l)^{2} \\
& Q(k, l)=Q_{0}+Q_{1} \frac{k+l}{2}+Q_{2}\left(\frac{k+l}{2}\right)^{3}+Q_{3}(k l)^{2} \\
& k_{i}=b_{i} / a_{i} l_{i}=c_{i} / a_{i}
\end{aligned}
$$

The model contains three parameters: $n, m$ and $p$. The optimal values of the exponents $m, p$ and $n$ are taken equal to $0.36,3$ and 1.30. $\left(P_{0}, P_{1}, P_{2}, P_{3}\right)$ and $\left(Q_{0}, Q_{1}, Q_{2}, Q_{3}\right)$ vector values can be found in [13].

Figure 4 compares the $K_{12}$ values based on Eqs. (§2) and denoted by $K_{12} p v$ with the $K_{12}$ values based on Eqs.28a-f and denoted by $K_{12}$ approx. The agreement between the two methods is very good. The relative standard deviation defined as $\sigma=\left(\sum_{i=1}^{N}\left(K_{12 a p p r o x}^{i} / K_{12 p v}^{i}-1\right)^{2} / N\right)^{1 / 2}$ is equal to 0.045

\section{2 inertia tensor of the resulting two-ellipsoid set}

For each collision event one may calculate the inertia tensor of the resulting body. After diagonalization, the semi-axes $\left(a=a_{12} ; b=b_{12} ; c=c_{12} ; a>b>c\right)$ of the equivalent ellipsoid are deduced. Hence, we get a data set about all the equivalent ellipsoids corresponding to two given ellipsoids colliding under shear flow. What are the relevant ellipsoid parameters if we are interested in the statistics from the data set? In our opinion, elongation or flattening is the most relevant: they may be defined as 
$b / a$ and $c / a$. Other asphericity parameters can be used [18,19]. An additional parameter characterizing the overall shape could be the semi-axis $a$ or the gyration radius $R_{g}$. In this section, we have selected the triplet $(a, b / a, c / a)$, which seems visually to be the most significant. Figure 5a,b,c shows the probability density function (pdf) for these parameters corresponding to all the collision events between two identical prolate spheroids $\left(a_{i}, b_{i}, c_{i}\right)=(1,0.2,0.2)$ having the same mass. The two methods developed in $\S 2$ and $\S 3$ are compared. It can be observed, that the agreement between them is very good in spite of the complex shape of pdf's.

In order to better understand the link between the colliding ellipsoids and the resulting equivalent ellipsoid, we have studied the collision between identical spheroids (prolate or oblate). The used method is as described in $\S 3$.

Figure $6 a, b, c$ represents the pdf of the largest semi-axis length, elongations b/a and c/a of the equivalent ellipsoid for colliding prolate spheroids $\left(a_{1}=1, b_{1}, b_{1}\right)$ with $b_{1} \in S=\{0.01 ; 0.1 ; 0.2 ; 0.5 ; 0.9\}$. Two colliding spheres $(1,1,1)$ lead to a prolate spheroid $(2.445,1,1)$. In this case, the pdf's of $a$ and $b / a$ are simply Dirac functions: the corresponding elongation value is 0.4085 . For the colliding prolate spheroids, the pdf's for $a$ and $b / a$ seem not to be dependent on the elongation $b_{1} / a_{1}$ of spheroids if the latter value is smaller than 0.2 . It is not the case for $c / a$ where the corresponding mean value follows the elongation value of the spheroids. The three pdf's are wide.

Figure $7 \mathrm{a}, \mathrm{b}, \mathrm{c}$ represents the pdf of the largest semi-axis length, elongations b/a and c/a of the equivalent ellipsoid for colliding oblate spheroids $\left(a_{1}=1,1, b_{1}\right)$ with $b_{1} \in S=\{0.01 ; 0.1 ; 0.2 ; 0.5 ; 0.9\}$. The pdf's for $a, b / a$ and $c / a$ seem not to be dependent on the elongation of spheroids if the latter value is smaller than 0.2 . b/a pdf for prolate spheroid and $c / a$ pdf for oblate spheroid look alike. 
Figure 8 represents the elongation parameters of the average equivalent ellipsoid against these of colliding identical spheroids. This shows that $b / a$ is relatively insensitive to the elongation parameters of the constitutive particles whereas $c / a$ values strongly depend on the colliding particle shapes. $b / a$ value is close to the one of a bisphere (=0.409).

Then, we have searched for an estimate of the three semi-axis lengths of the average equivalent ellipsoid, as already achieved for the collision rate constant (sub-section 4.1). The same set of colliding ellipsoids has been selected. The effect of the internal density of the ellipsoids has also been considered. For this purpose, the density $\rho_{1}$ of the ellipsoid 1 is taken equal to 1 whereas the density $\rho_{2}$ of ellipsoid 2 is taken within the range $\mathrm{W}$ :

$\rho_{2} \in W=\{0.02 ; 0.1 ; 0.2 ; 0.5 ; 1 ; 2 ; 5 ; 10 ; 20\}$

Taking the value one for $a_{1}$ means that all the semi-axis lengths of the average equivalent ellipsoid will be made dimensionless by $a_{1}$. Taking the value one for $\rho_{1}$ has no effect on the length parameters of the average equivalent ellipsoid. Only the density ratio $\rho_{2} / \rho_{1}$ is relevant. Accordingly, 12150 different pairs of colliding ellipsoids have been studied.

First, the effect of the MC run number $N_{r}$ on the accuracy of ellipsoid parameters has been studied. Considering two extreme $N_{r}$ values $\left(N_{r}=210^{4}\right.$ and $\left.10^{6}\right)$, the relative deviations between the calculated ellipsoid parameters are $1.8 \%$ for $a, 2.9 \%$ for $b$, $3.8 \%$ for $c$. Therefore, a correct estimate of the ellipsoid parameters can be obtained by strongly reducing the $\mathrm{MC}$ run number while also reducing the computation time. However, it must be underlined that the calculation fails for a few pairs $(0.25 \%$ of the total pair number); The reason is simply that, for certain pair, no collision occurs within $210^{4}$ random possible events. 
The data file corresponding to the 12150 pairs of colliding ellipsoids (with $N_{r}=210^{4}$ ) will be provided upon request.

The approximate expressions for the ellipsoid parameters must obey the following requirements:

- symmetrical with respect to the two colliding ellipsoids

- give the parameters of the ellipsoid with the largest mass if the collision happens between two ellipsoids with very different mass values

- match the analytical expressions available for certain ellipsoid pairs, e.g. for sphere pairs

To be compatible with the physics of the collision, we start from an expression containing the diagonalized inertia tensor:

$$
\mathbf{D}_{\mathbf{E}, \mathbf{1 2}}=\mathbf{D}_{\mathbf{E}, \mathbf{1}}+\mathbf{D}_{\mathbf{E}, \mathbf{2}}+m_{1} m_{2} /\left(m_{1}+m_{2}\right) \mathbf{f}_{12}
$$

or

$b^{2}+c^{2}=\left(b_{1}^{2}+c_{1}^{2}\right) \phi_{1}+\left(b_{2}^{2}+c_{2}^{2}\right) \phi_{2}+5 \phi_{1} \phi_{2} f_{b c}$

$a^{2}+c^{2}=\left(a_{1}^{2}+c_{1}^{2}\right) \phi_{1}+\left(a_{2}^{2}+c_{2}^{2}\right) \phi_{2}+5 \phi_{1} \phi_{2} f_{a c}$

$a^{2}+b^{2}=\left(a_{1}^{2}+b_{1}^{2}\right) \phi_{1}+\left(a_{2}^{2}+b_{2}^{2}\right) \phi_{2}+5 \phi_{1} \phi_{2} f_{a b}$

with $\phi_{i}=m_{i} /\left(m_{1}+m_{2}\right) . f_{b c}, f_{a c}, f_{a b}$ are to be determined. In the case of sphere pairs, we have:

$f_{b c}=0, f_{a c}=f_{a b}=\left(R_{1}+R_{2}\right)^{2}$

We have assumed that $f_{b c}, f_{a c}, f_{a b}$ have a quadratic form in six variables:

$\mathbf{u}=\left(u_{1}, u_{2}, u_{3}, u_{4}, u_{5}, u_{6}\right)=\left(a_{1}, b_{1}, c_{1}, a_{2}, b_{2}, c_{2}\right)$

i.e. $f_{b c}={ }^{T} \mathbf{u} \mathbf{F}_{\mathbf{b c}} \mathbf{u}$

The $6 \times 6$ matrix $\mathbf{F}_{\mathbf{b c}}$ is symmetrical. Then, considering the invariance of $f_{b c}$ by exchanging some variables, we obtain the reduced form: 
$f_{b c}=C_{1} \sum_{i=1}^{6} u_{i}^{2}+2 C_{2}\left(u_{1} u_{2}+u_{1} u_{3}+u_{2} u_{3}\right)+2 C_{2}\left(u_{4} u_{5}+u_{4} u_{6}+u_{5} u_{6}\right)+2 \sum_{\substack{1 \leq i \leq 3 \\ 4 \leq j \leq 6}} C_{i, j} u_{i} u_{j}$

This is also valid for $f_{a c}$ and $f_{a b}$, but with different values for $C_{1}, C_{2}, C_{i, j}$.

For two unlike spheres, i.e. $\mathbf{u}=\left(R_{1}, R_{1}, R_{1}, R_{2}, R_{2}, R_{2}\right)$, we have:

$$
\begin{aligned}
& f_{b c}:\left(R_{1}^{2}+R_{2}^{2}\right)\left(3 C_{1}+6 C_{2}\right)+2 R_{1} R_{2} \sum_{\substack{1 \leq i \leq 3 \\
4 \leq j \leq 6}} C_{i, j}=0 \quad \forall R_{1}, R_{2} \\
& f_{a c}, f_{a b}:\left(R_{1}^{2}+R_{2}^{2}\right)\left(3 C_{1}+6 C_{2}-1\right)+2 R_{1} R_{2}\left(\sum_{\substack{1 \leq i \leq 3 \\
4 \leq j \leq 6}} C_{i, j}-1\right)=0 \quad \forall R_{1}, R_{2}
\end{aligned}
$$

As a consequence, $3 C_{1}+6 C_{2}=\delta ; \quad \sum_{\substack{1 \leq i \leq 3 \\ 4 \leq j \leq 6}} C_{i, j}=\delta$ with $\delta=0$ for $\mathrm{f}_{\mathrm{bc}}\left(=\mathrm{f}_{0}\right)$ and $\delta=1$ for $\mathrm{f}_{\mathrm{ac}}\left(=\mathrm{f}_{1}\right)$ and $\mathrm{f}_{\mathrm{ab}}\left(=\mathrm{f}_{1}\right)$.

Finally, we have to determine 9 unknown parameters for each of three f:

$$
\begin{gathered}
f_{\delta}=C_{1}\left(\sum_{i=1}^{6} u_{i}^{2}\right)+\left(\delta / 3-C_{1}\right)\left(u_{1} u_{2}+u_{1} u_{3}+u_{2} u_{3}+u_{4} u_{5}+u_{4} u_{6}+u_{5} u_{6}\right) \\
+2 \delta u_{1} u_{4}+2 \sum_{\substack{1 \leq i \leq 3 \\
4 \leq j \leq 6 \\
(i, j) \neq(1,4)}} C_{i, j}\left(u_{i} u_{j}-u_{1} u_{4}\right)
\end{gathered}
$$

An optimization procedure has been used for determining the $C$-parameters: optimization calculations were performed by considering the ratio between the elements of the diagonalized inertia tensor coming from approximation and those issued from Monte-Carlo simulations. Table 1 contains the optimized parameters in Eq.36.

Then, from the diagonal elements of approximate $\mathbf{D}_{\mathbf{E}, \mathbf{1 2}}$ (Eq. 29) the parameters $a, b, c$ of the average equivalent ellipsoid have been calculated. The relative standard deviation between the values from approximate expression and the MC calculation was found to be equal to 0.015 , 0.148, 0.197 for $a, b$ and $c$, respectively. Excluding collisions between ellipsoids with the smallest $b / a$ and $c / a$ parameters $(=0.01)$ leads to the following relative standard deviation: 
$0.013,0.054,0.12$ for $a, b$ and $c$, respectively. However this reduced ellipsoid set includes the expected equivalent ellipsoid formed during aggregation, making the approximation suitable for this application.

\section{3 kernel for collision between two sphere clusters}

We consider two types of clusters:

i) ordered clusters consisting of chains of $(2,10,50)$ identical spheres (with radius value equal to 1$)$, square $(2 \times 2,4 \times 4,7 \times 7,4 \times 7)$ of touching spheres, parallelepiped $(2 \times 2 \times 2,4 \times 4 \times 4,5 \times 5 \times 2)$ of spheres. These packed clusters are dense and have edges and corners.

ii) random clusters coming from the simulation of the shear aggregation between smaller aggregates using a Monte Carlo method. These clusters must represent more realistically the aggregates during the aggregation of spheres. Ten clusters with the sphere number within the range $[7 ; 52]$ were selected. These clusters are loose and have a solid volume fraction higher than 0.1 .

Then we have considered the collision between any pair of ordered clusters and between any pair of random clusters. We have calculated the collision rate constant (one hundred values for ordered clusters and one hundred values for random clusters) for all these pairs. $N_{r}$ is taken equal to $10^{5}$. The $K_{12}$ range for ordered clusters is within [30; 20000], whereas the $K_{12}$ range for random clusters is narrower, i.e. [300; 6000]. Each (structured or random) collider is characterized either by the coordinates of the constituting spheres or by the $(a, b, c)$ parameters of the equivalent ellipsoid or more simply by the gyration radius. According to the geometrical parameters depicting the collider, $K_{12}$ values are calculated by MCS for sphere clusters (see $\$ 3$ ), by MCS for 
ellipsoids (§3), and by considering their gyration radius into the Smoluchowski’s equation:

$$
K_{12, S}=\frac{4}{3} \dot{\gamma} \alpha^{3}\left(R_{g, 1}+R_{g, 2}\right)^{3}
$$

The $\alpha$ coefficient is chosen so that $\alpha R_{g}=R$ for a sphere with the radius $R$, i.e $\alpha=\sqrt{5 / 3}$.

The figure 9 represents the $K_{12}$ (named $K_{12, E E}$ ) values calculated for equivalent ellipsoids and from Smoluchowski equation (named $K_{12, S}$ ) versus $K_{12}$ values for sphere clusters ( named $K_{12, C C}$ ). It can be observed that the correlation between $K_{12, E E}$ and $K_{12, C C}$ is good over all the $K_{12}$ range for structured and random clusters, whereas this correlation fits less well with Smoluchowski's approximation for the two types of clusters, and especially badly for the large $K_{12}$ values of ordered clusters. This last discrepancy strictly corresponds to the collision of the largest sphere chains with other clusters. Finally, we may write:

$$
K_{12, E E} / K_{12, C C}=1.27 \pm 0.16
$$

The collision rate constant calculated from the equivalent ellipsoid is always a little larger than the one derived from cluster. This will be discussed below.

\section{4 pdf of geometrical parameters for cluster collision}

The pdf of geometrical parameters for the resulting cluster after each cluster collision event have been determined. We have considered the collision of two sphere clusters and alternatively the collision of their equivalent ellipsoids. After $10^{7} \mathrm{MC}$ runs, the pdf of $a, b, c$ parameters of the resulting body have been calculated. These simulations have been performed for several pairs of ordered and random clusters. For instance, the figure 10a represents the pdf's for $a, b, c$ for the collision of two random clusters (see 
figure 10c), whereas the pdf's for $b / a$ and $c / a$ for the same collision events are presented on figure 10b. It can be observed that the collisions of sphere clusters and their equivalent ellipsoids lead to the nearby pdf's of parameters of the resulting body. A finer analysis shows that $b, c$ pdf's are very close while $a$ pdf's are slightly offset. The $a$ pdf of the resulting body coming from ellipsoids collision is shifted to the large size values. These results are valid for all sphere cluster pairs studied. It may be confirmed by calculating the $a, b, c$ parameters of the average equivalent ellipsoid for EE collisions and CC collisions:

$$
a_{12, E E} / a_{12, C C}=1.09 \pm 0.03 \quad b_{12, E E} / b_{12, C C}=1 \pm 0.01 \quad c_{12, E E} / c_{12, C C}=1 \pm 0.01
$$

And

$$
R_{g_{12, E E}} / R_{g_{12, C C}}=1.075 \pm 0.02
$$

At this level two comments may be made:

- The result concerning the parameters of the average equivalent ellipsoid is conform with this for the collision rate constant (Eq.38) as the collision rate constant is roughly proportional to the cubic gyration radius.

- We may state that the collision between two sphere clusters is well represented by the collision of their equivalent ellipsoids. As a consequence of the previous point, the fit will be improved if the largest semi-axis of the average equivalent ellipsoid of the resulting body is reduced by a factor 1.09 independently of the pair of colliding clusters. This point will be particularly significant when studying the whole aggregation process of primary particles, since a small deviation of the geometrical parameters will be amplified during the reiteration of the collision act.

\section{Conclusion}


In this paper we have presented two methods of calculating the parameters corresponding to the collision and aggregation of two ellipsoids. These two methods produce very close results and therefore ensure the reliability of the generated data. The collision rate constant and the statistics concerning the geometrical parameters of the equivalent ellipsoid of the aggregate have been determined and checked. On the other hand, we have shown that replacing sphere clusters by their equivalent ellipsoids leads to a good representation of the collision events. Empirical expressions have been proposed for the collision rate constant and the $a, b, c$ parameters of the average equivalent ellipsoid. However, it must be noted that the smallest parameter, i.e. $c$, may be poorly valued by our approximation for a few colliding ellipsoid set. These expressions make much faster the use of the basic aggregation act if the particles are depicted by their mass content and the $a, b, c$ parameters. They could therefore be used in a multivariate population balance modelling for particle aggregation.

\section{References}

[1] R. Jullien and R. Botet, “Aggregation and Fractal Aggregates”, 1987, World Scientific Publishing

[2] M. Elimelech, J. Gregory, X. Jia, R. Williams, “Particle deposition \& aggregation”, Butterworth-Heinemann, 1995

[3] G. Pranami, M.H. Lamm, R.D. Vigil, "Molecular dynamics simulation of fractal aggregate diffusion”, Physical Review E82, 051402 (2010)

[4] F.E. Torres, W.B. Russel, W.R. Schowalter, "Simulations of coagulation in viscous flows", J. of colloid and Interface Science 145(1991)51-73 
[5] G. Frungieri and M. Vanni, "Shear - induced aggregation of colloidal particles: A comparison between two different approaches to the modelling of colloidal interactions", Canadian Journal of Chemical Engineering, 95(2017), 1768-1780

[6] A. Cameirao, R. David, F. Espitalier, F. Gruy, Journal of Crystal Growth, 310(2008) 4152-4162.

[7] F. Gruy, "Inertia tensor as morphological descriptor for aggregation dynamics", Colloids and Surfaces A: Physicochemical and Engineering Aspects, 482(2015)154-164.

[8] J.S. Marshall and S. Li, “Adhesive Particle Flow: A Discrete-Element Approach”,2014, Cambridge University Press

[9] M. Smith, T. Matsoukas, “Constant-number Monte Carlo simulation of population balances”, Chem. Eng. Sci., 53(1998)1777

[10] «Euler angles », Wikipedia, the free encyclopedia

[11] E.W. Weisstein, "Euler Angles." From MathWorld--A Wolfram Web Resource. http://mathworld.wolfram.com/EulerAngles.html

[12] L. Landau et E. Lifchitz, Mécanique, Editions MIR, Moscou, 1969

[13] F. Gruy and P. Nortier, "Collision rate constant for non-spherical particles moving under shear flow”, Colloids and Surfaces A: Physicochem. Eng. Aspects 522 (2017) 552562

[14] G.K. Batchelor, J.T. Green, “The hydrodynamic interaction of two small freelymoving spheres in a linear flow field”, J. Fluid Mech. 56(1972)375-400

[15] D.J. Jeffrey, Y. Onishi, "Calculation of the resistance and mobility functions for two unequal rigid spheres in low Reynolds number flow”, J. Fluid Mech. 139(1984)261-290

[16] C. Walck, Handbook on statistical distributions for experimentalists, internal report SUF-PFY, Stockholm, 2007 
[17] E.L. Rees, "Graphical Discussion of the Roots of a Quartic Equation", The American Mathematical Monthly. 29 (1922) 51-55.

[18] J. Rudnick, G. Gaspari, "The asphericity of random walks", J. Phys. A: Math. Gen. 19(1986)L191-193

[19] J. Rudnick, G. Gaspari, “The shapes of random walks”, Science, 237(1987) p384-389 


\section{Annex 1: calculation of $\partial \mathbf{b} / \partial \psi_{q}$ with $q=y, z$}

Starting from the definition of the $\mathbf{b}$ vector:

$\mathbf{b}=\frac{\mathbf{A}^{-1} \mathbf{u}}{\sqrt{\mathbf{u}^{\mathrm{T}} \mathbf{A}^{-1} \mathbf{u}}}$

Its derivation leads to:

$$
\frac{\partial \mathbf{b}}{\partial \psi_{q}}=\frac{1}{\left(\mathbf{u}^{\mathbf{T}} \mathbf{A}^{-1} \mathbf{u}\right)^{3 / 2}}\left[\left(\mathbf{u}^{\mathbf{T}} \mathbf{A}^{-1} \mathbf{u}\right)\left(\mathbf{A}^{-1} \frac{\partial \mathbf{u}}{\partial \psi_{q}}\right)-\frac{1}{2}\left(\mathbf{u}^{\mathbf{T}} \mathbf{A}^{-1} \frac{\partial \mathbf{u}}{\partial \psi_{q}}+\frac{\partial \mathbf{u}^{\mathbf{T}}}{\partial \psi_{q}} \mathbf{A}^{-1} \mathbf{u}\right)\left(\mathbf{A}^{-1} \mathbf{u}\right)\right]
$$

Let us first remark that, given two matrices $\mathbf{M}$ and $\mathbf{N}$ and three vectors $\mathbf{a}, \mathbf{b}$ and $\mathbf{c}$ :

$$
(\mathbf{M a})\left(\mathbf{b}^{\mathrm{T}} \mathbf{N c}\right)=\mathbf{M}\left(\mathbf{a b}^{\mathrm{T}}\right) \mathbf{N c}
$$

$\mathbf{a b}^{\mathbf{T}}$ is the outer or dyadic product of $\mathbf{a}$ and $\mathbf{b}$ vectors.

As $\mathbf{A}_{\mathbf{E}}$ is a diagonal matrix, $\mathbf{A}^{-\mathbf{1}}$ is symmetrical and

$$
\left(\mathbf{A}^{-1} \mathbf{u}\right)^{\mathrm{T}}=\mathbf{u}^{\mathrm{T}} \mathbf{A}^{-1}
$$

As the product of matrices commutes with the multiplication by a scalar and using (A-2):

$$
\begin{aligned}
& \left(\mathbf{u}^{\mathbf{T}} \mathbf{A}^{-1} \mathbf{u}\right)\left(\mathbf{A}^{-1} \frac{\partial \mathbf{u}}{\partial \psi_{q}}\right)=\left(\mathbf{A}^{-1} \frac{\partial \mathbf{u}}{\partial \psi_{q}}\right)\left(\mathbf{u}^{\mathbf{T}} \mathbf{A}^{-1} \mathbf{u}\right)=\mathbf{A}^{-1}\left(\frac{\partial \mathbf{u}}{\partial \psi_{q}} \mathbf{u}^{\mathbf{T}}\right) \mathbf{A}^{-1} \mathbf{u} \\
& \left(\frac{\partial \mathbf{u}^{\mathbf{T}}}{\partial \psi_{q}} \mathbf{A}^{-1} \mathbf{u}\right)\left(\mathbf{A}^{-1} \mathbf{u}\right)=\left(\mathbf{A}^{-1} \mathbf{u}\right)\left(\frac{\partial \mathbf{u}^{\mathbf{T}}}{\partial \psi_{q}} \mathbf{A}^{-1} \mathbf{u}\right)=\mathbf{A}^{-1}\left(\mathbf{u} \frac{\partial \mathbf{u}^{\mathbf{T}}}{\partial \psi_{q}}\right) \mathbf{A}^{-1} \mathbf{u}
\end{aligned}
$$

As the transpose of a scalar is itself and as $\mathbf{A}^{\mathbf{- 1}}$ is symmetrical:

$$
\left(\mathbf{u}^{\mathbf{T}} \mathbf{A}^{-1} \frac{\partial \mathbf{u}}{\partial \psi_{q}}\right)\left(\mathbf{A}^{-1} \mathbf{u}\right)=\left(\mathbf{A}^{-1} \mathbf{u}\right)\left(\mathbf{u}^{\mathbf{T}} \mathbf{A}^{-1} \frac{\partial \mathbf{u}}{\partial \psi_{q}}\right)=\left(\mathbf{A}^{-1} \mathbf{u}\right)\left(\frac{\partial \mathbf{u}^{\mathbf{T}}}{\partial \psi_{q}} \mathbf{A}^{-1} \mathbf{u}\right)=\mathbf{A}^{-1}\left(\mathbf{u} \frac{\partial \mathbf{u}^{\mathbf{T}}}{\partial \psi_{q}}\right) \mathbf{A}^{-1} \mathbf{u} \quad \text { A-6 }
$$

So that:

$$
\frac{\partial \mathbf{b}}{\partial \psi_{q}}=\frac{1}{\left(\mathbf{u}^{\mathbf{T}} \mathbf{A}^{-1} \mathbf{u}\right)^{3 / 2}} \mathbf{A}^{-1} \mathbf{D}_{\mathbf{q}} \mathbf{A}^{-1} \mathbf{u}
$$

With 
$\mathbf{D}_{q}=\frac{\partial \mathbf{u}}{\partial \psi_{q}} \mathbf{u}^{\mathbf{T}}-\mathbf{u} \frac{\partial \mathbf{u}^{\mathbf{T}}}{\partial \psi_{q}}$

Considering the definition of $\mathbf{u}$ and the rotation matrix:

$$
\mathbf{u}=\left(\begin{array}{c}
\cos \left(\psi_{y}\right) \cos \left(\psi_{z}\right) \\
\sin \left(\psi_{z}\right) \\
-\sin \left(\psi_{y}\right) \cos \left(\psi_{z}\right)
\end{array}\right) \quad \mathbf{R}_{\Psi}=\left(\begin{array}{ccc}
\cos \left(\psi_{y}\right) \cos \left(\psi_{z}\right) & -\cos \left(\psi_{y}\right) \sin \left(\psi_{z}\right) & \sin \left(\psi_{y}\right) \\
\sin \left(\psi_{z}\right) & \cos \left(\psi_{z}\right) & 0 \\
-\sin \left(\psi_{y}\right) \cos \left(\psi_{z}\right) & \sin \left(\psi_{y}\right) \sin \left(\psi_{z}\right) & \cos \left(\psi_{y}\right)
\end{array}\right)
$$

A simple but rather cumbersome calculation yields:

$$
\begin{aligned}
& D_{\mathbf{y}}=\left(\begin{array}{ccc}
0 & -\sin \left(\psi_{y}\right) \sin \left(\psi_{z}\right) \cos \left(\psi_{z}\right) & \cos ^{2}\left(\psi_{z}\right) \\
\sin \left(\psi_{y}\right) \sin \left(\psi_{z}\right) \cos \left(\psi_{z}\right) & 0 & \cos \left(\psi_{y}\right) \sin \left(\psi_{z}\right) \cos \left(\psi_{z}\right) \\
-\cos ^{2}\left(\psi_{z}\right) & -\cos \left(\psi_{y}\right) \sin \left(\psi_{z}\right) \cos \left(\psi_{z}\right) & 0
\end{array}\right) \text { A-9 } \\
& D_{\mathbf{z}}=\left(\begin{array}{ccc}
0 & -\cos \left(\psi_{y}\right) & 0 \\
\cos \left(\psi_{y}\right) & 0 & -\sin \left(\psi_{y}\right) \\
0 & \sin \left(\psi_{y}\right) & 0
\end{array}\right)
\end{aligned}
$$




\section{List of figures}

Figure 1: orthonormal system and velocity gradient

Figure 2: collision between two objects: the object 1 with a given orientation collides with the object 2 at two different points.

Figure 3: $K_{12} \mathrm{proj} / K_{12} p v$ ratio versus $K_{12} p v$

Figure 4: $K_{12}$ approx $/ K_{12} p v$ ratio versus $K_{12} p v$

Figure 5a: pdf corresponding to the largest semi-axis of the equivalent ellipsoid. Collision between $(1,0.2,0.2)$ and $(1,0.2,0.2)$. o, red: issued from method $\S 2 ; *$, black: issued from method $\S 3$

Figure 5b: pdf corresponding to the largest elongation $b / a$. Collision between $(1,0.2,0.2)$ and $(1,0.2,0.2)$. o, red: issued from method $\S 2 ; *$, black: issued from method $\S 3$

Figure 5c: pdf corresponding to the smallest elongation $c / a$. Collision between $(1,0.2,0.2)$ and $(1,0.2,0.2)$. o, red: issued from method $\S 2 ; *$, black: issued from method $\S 3$

Figure 6a, b, c: Collision of two identical prolate spheroids $\left(1, b_{1}, b_{1}, m_{1}=1\right)$ with $b_{1} \in S=\{0.01 ; 0.1 ; 0.2 ; 0.5 ; 0.9\} ; b_{1}=0.9-$ black $; b_{1}=0.5+$ green $; b_{1}=0.2$ o red $; b_{1}=0.1 *$ blue; $b_{1}=0.01 \times$ magenta
a) pdf for $a$
b) pdf for $b / a$
c) pdf for $c / a$

Figure 7a, b, c: Collision of two identical oblate spheroids $\left(1,1, b_{1}, m_{1}=1\right)$ with $b_{1} \in S=\{0.01 ; 0.1 ; 0.2 ; 0.5 ; 0.9\} ; b_{1}=0.9$ - black $; b_{1}=0.5+$ green; $b_{1}=0.2$ o red $; b_{1}=0.1 *$ blue; $b_{1}=0.01 \times$ magenta
a) pdf for $a$
b) pdf for $b / a$
c) pdf for $c / a$

Figure 8: Collision of two identical prolate or oblate spheroids: elongation parameters of the average equivalent ellipsoid 
Spheroids $\left(1,1, b_{1}, m_{1}=1\right)$ solid line; $\left(1, b_{1}, b_{1}, m_{1}=1\right)$ dashed line with

$b_{1} \in S=\{0.01 ; 0.1 ; 0.2 ; 0.5 ; 0.9\}$

Average equivalent ellipsoids: black curve: $b / a$; red curve: $c / a$

Figure 9: $K_{12, E E}(+)$ and $K_{12, S}\left(\right.$ o) versus $K_{12, C C}$. Black marker corresponds to ordered clusters; red marker corresponds to random clusters. The solid line corresponds to $\mathrm{y}=1.27 \mathrm{x}$

Figure 10a: pdf $n$ for $a, b, c$ parameters of equivalent ellipsoid for the collision of two random sphere clusters (A and B). $a$ : solid line; $b$ : dashed line; $c$ : dash-dot line.

Statistics from collisions between sphere clusters: red; Statistics from collisions between equivalent ellipsoids of clusters: black.

Figure 10b: pdf $n$ for $b / a$ and $c / a$ parameters of equivalent ellipsoid for the collision of two random sphere clusters (A and B). b/a: dashed line ; $c / a$ : solid line.

Statistics from collisions between sphere clusters: red; Statistics from collisions between equivalent ellipsoids of clusters.

Figure 10c: Sphere cluster A (N=52) left sphere cluster $B(N=17)$ right 

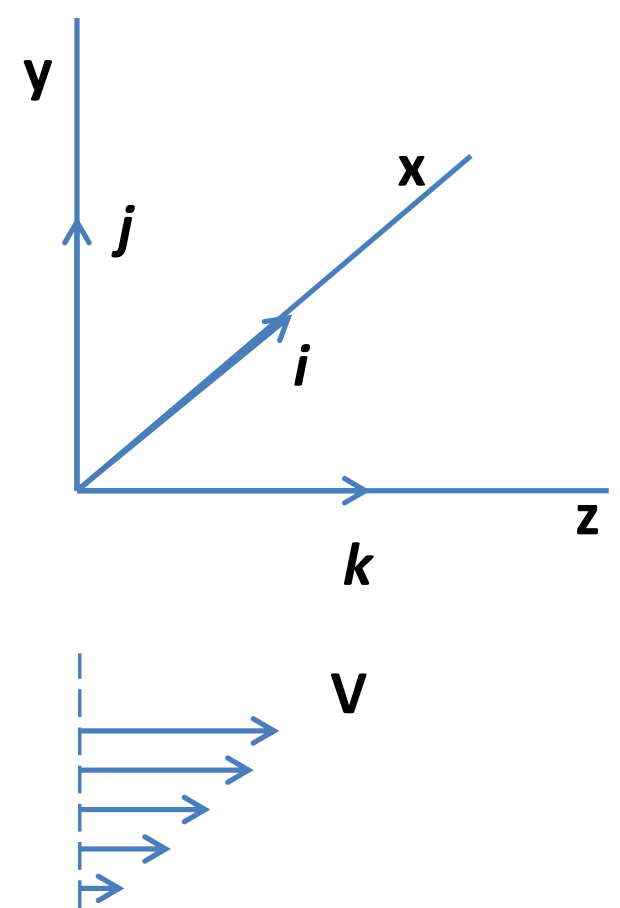

Figure 1: orthonormal system and velocity gradient 


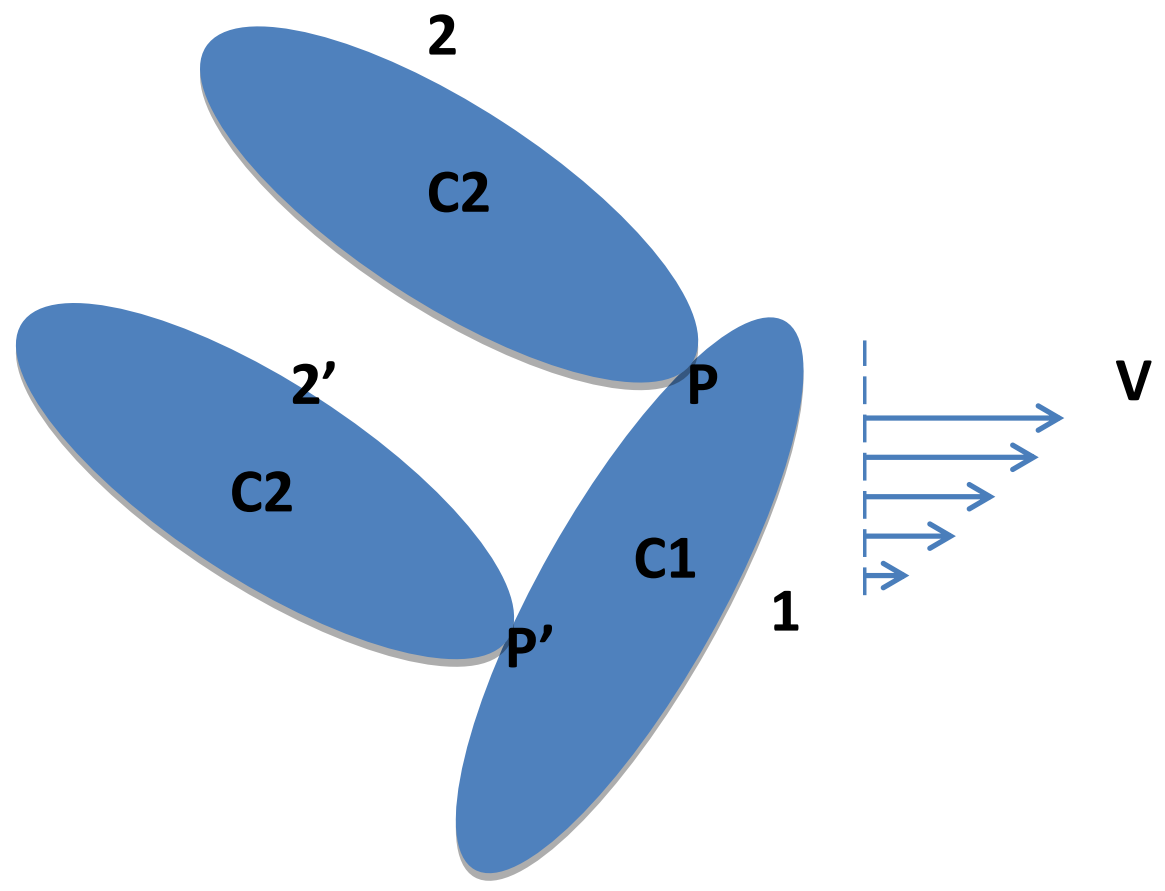

Figure 2: collision between two objects: the object 1 with a given orientation collides with the object 2 at two different points. 


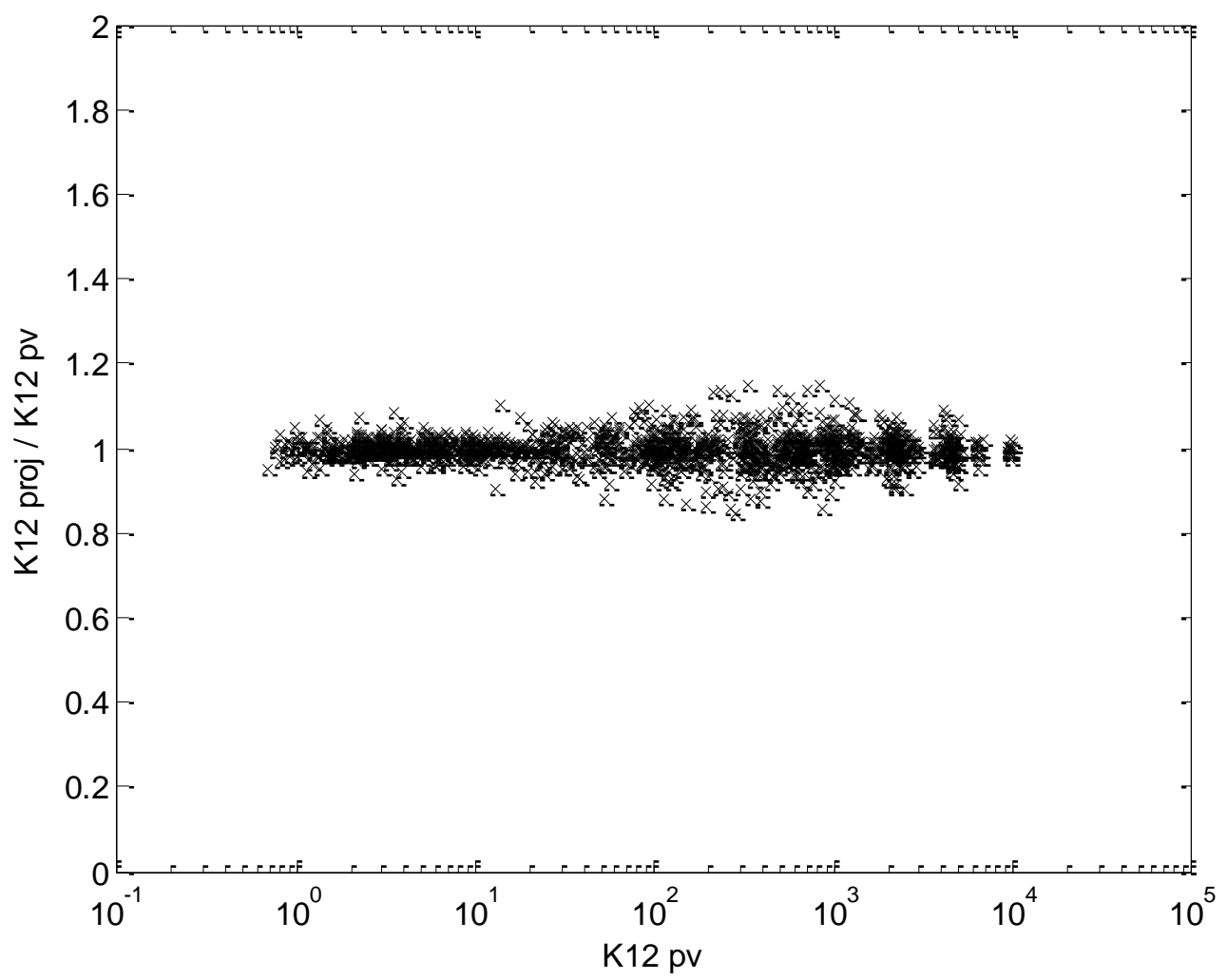

Figure 3: $K_{12} \mathrm{proj} / K_{12} p v$ ratio versus $K_{12} p v$ 


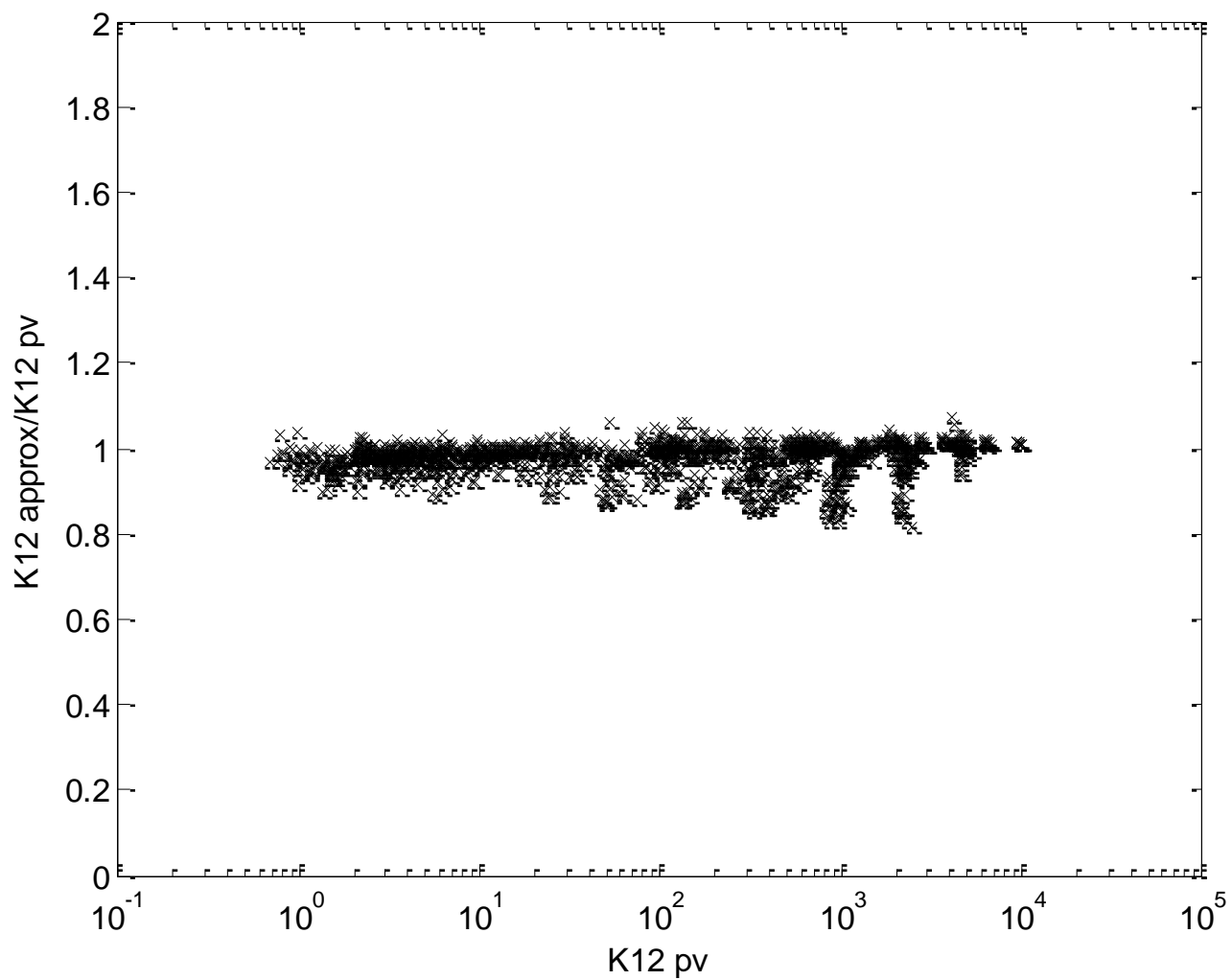

Figure 4: $K_{12}$ approx $/ K_{12} p v$ ratio versus $K_{12} p v$ 


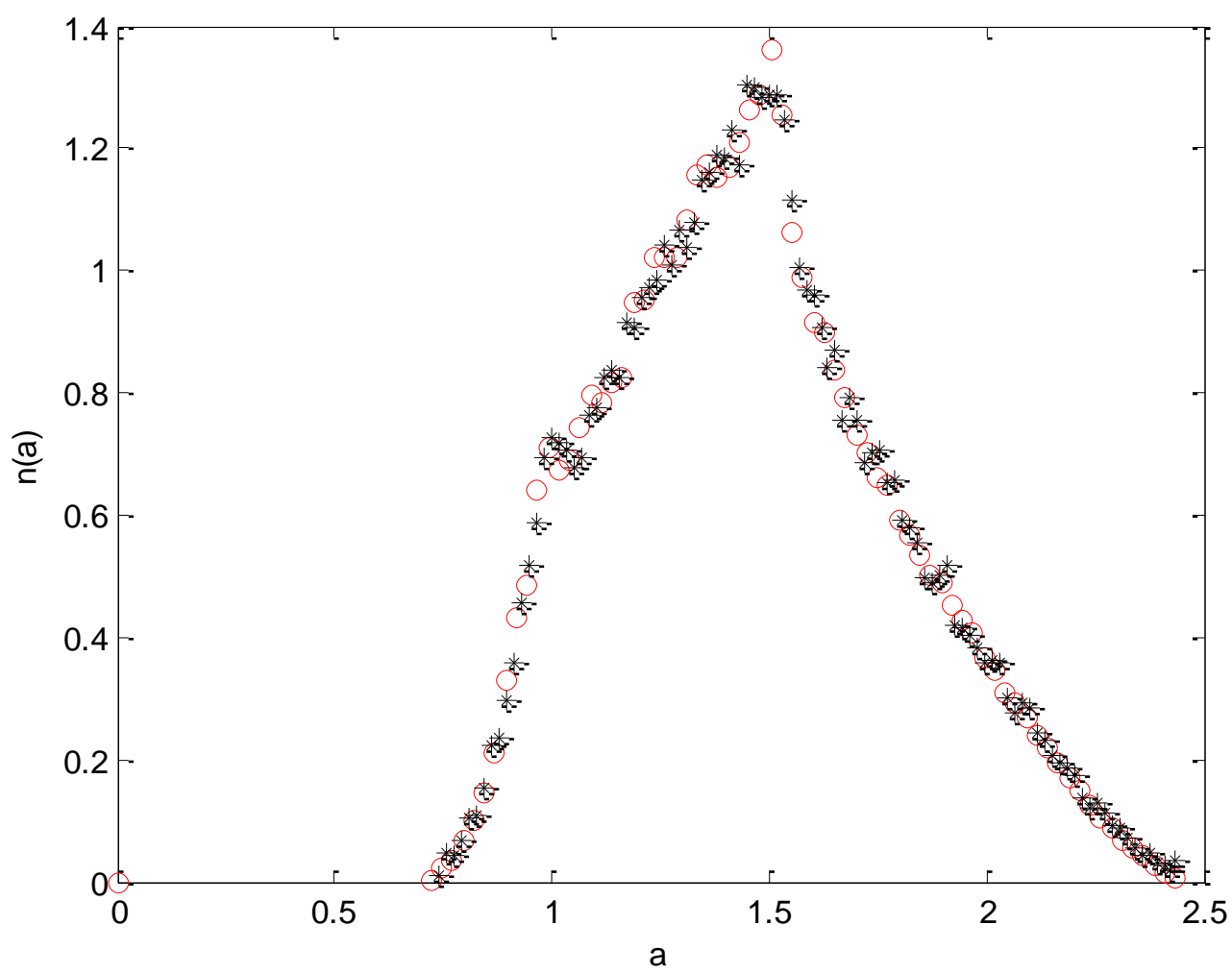

Figure 5a: pdf corresponding to the largest semi-axis of the equivalent ellipsoid. Collision between $(1,0.2,0.2)$ and $(1,0.2,0.2)$. o, red: issued from method $\S 2 ; *$, black: issued from method $\S 3$ 




Figure 5b: pdf corresponding to the largest elongation b/a. Collision between $(1,0.2,0.2)$ and $(1,0.2,0.2)$. o, red: issued from method $\S 2 ; *$, black: issued from method $\S 3$ 




Figure 5c: pdf corresponding to the smallest elongation $c / a$. Collision between $(1,0.2,0.2)$ and $(1,0.2,0.2)$. o, red: issued from method $\S 2 ; *$, black: issued from method $\S 3$ 


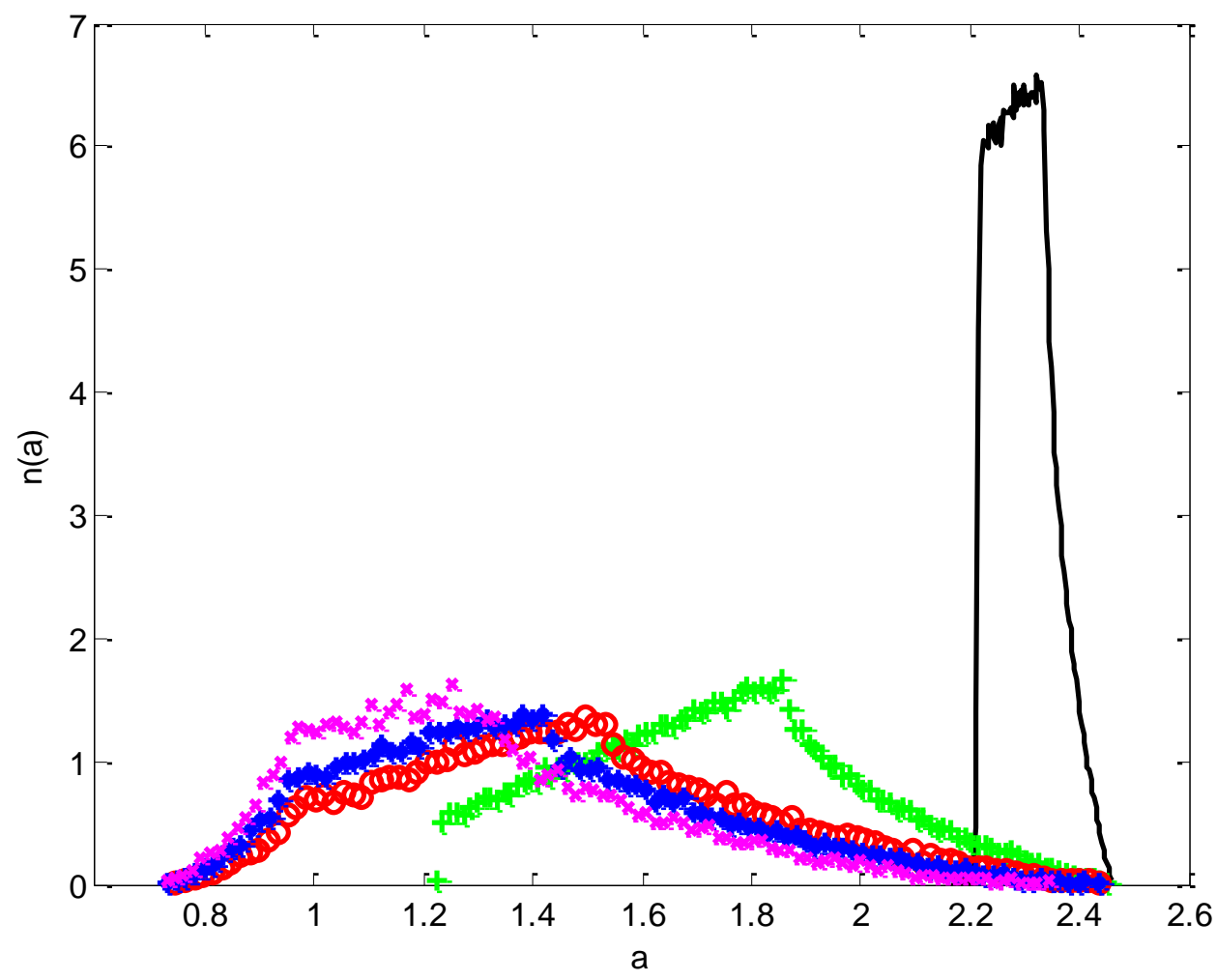

Figure 6a, b, c: Collision of two identical prolate spheroids $\left(1, b_{1}, b_{1}, m_{1}=1\right)$ with $b_{1} \in S=\{0.01 ; 0.1 ; 0.2 ; 0.5 ; 0.9\} ; b_{1}=0.9$ - black $; b_{1}=0.5+$ green $; b_{1}=0.2$ o red; $b_{1}=0.1 *$ blue; $b_{1}=0.01 \times$ magenta

Figure 6a: pdf for $a$ 


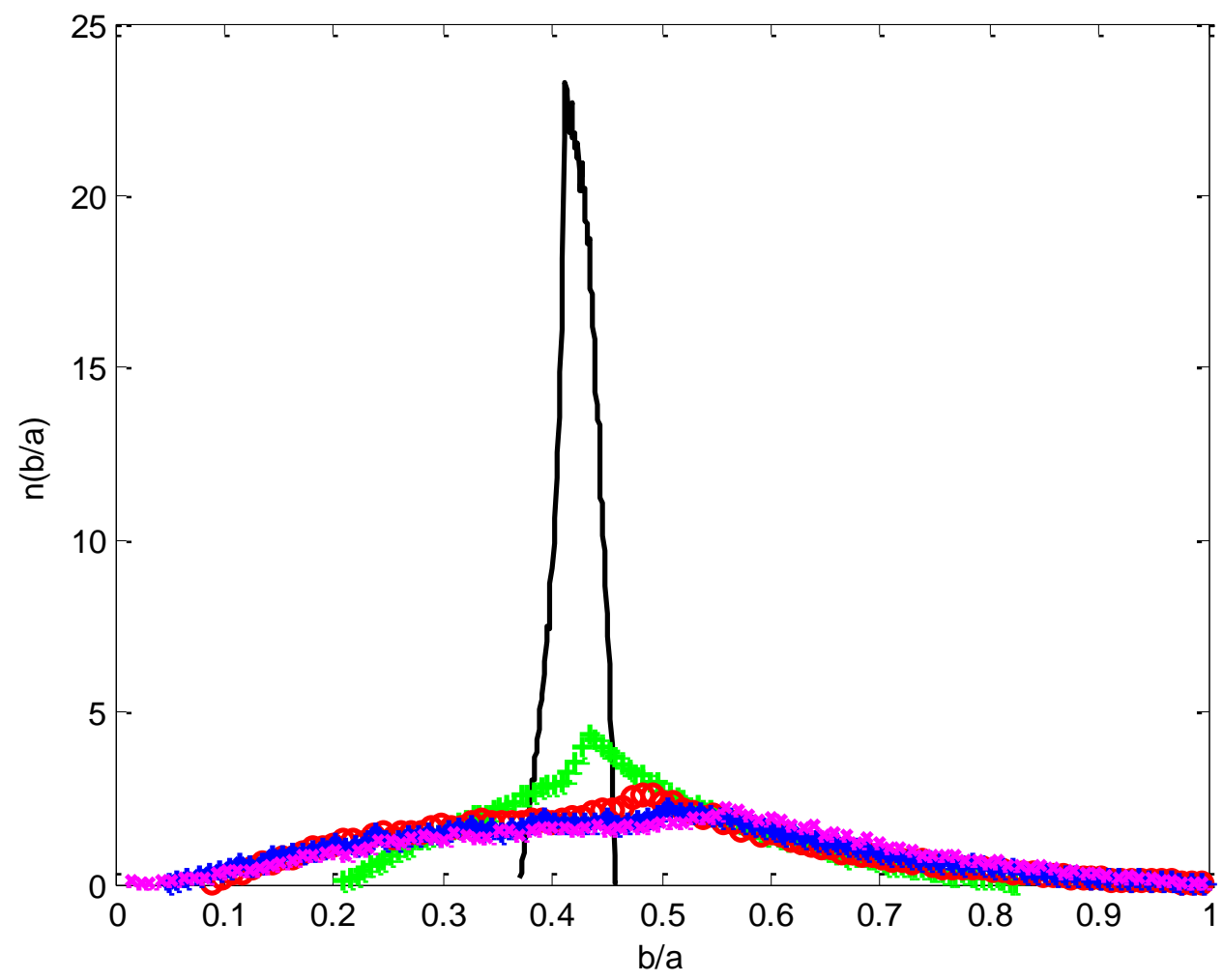

Figure 6b: pdf for $b / a$ 


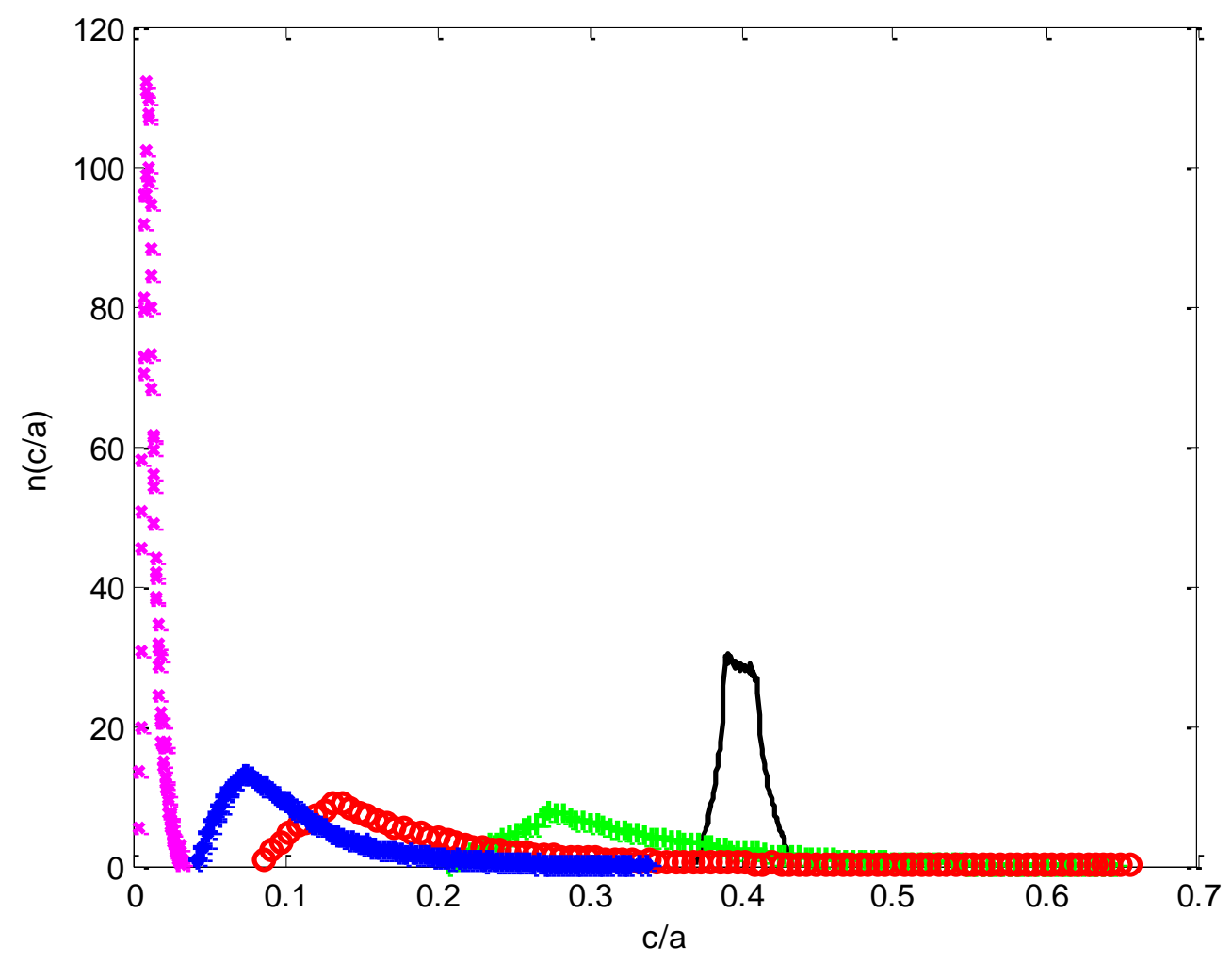

Figure 6c: pdf for $c / a$ 
Figure 7a, b, c: Collision of two identical oblate spheroids $\left(1,1, b_{1}, m_{l}=1\right)$ with $b_{1} \in S=\{0.01 ; 0.1 ; 0.2 ; 0.5 ; 0.9\} ; b_{1}=0.9$ - black $; b_{1}=0.5+$ green $; b_{1}=0.2$ o red $; b_{1}=0.1 *$ blue; $b_{1}=0.01 \times$ magenta

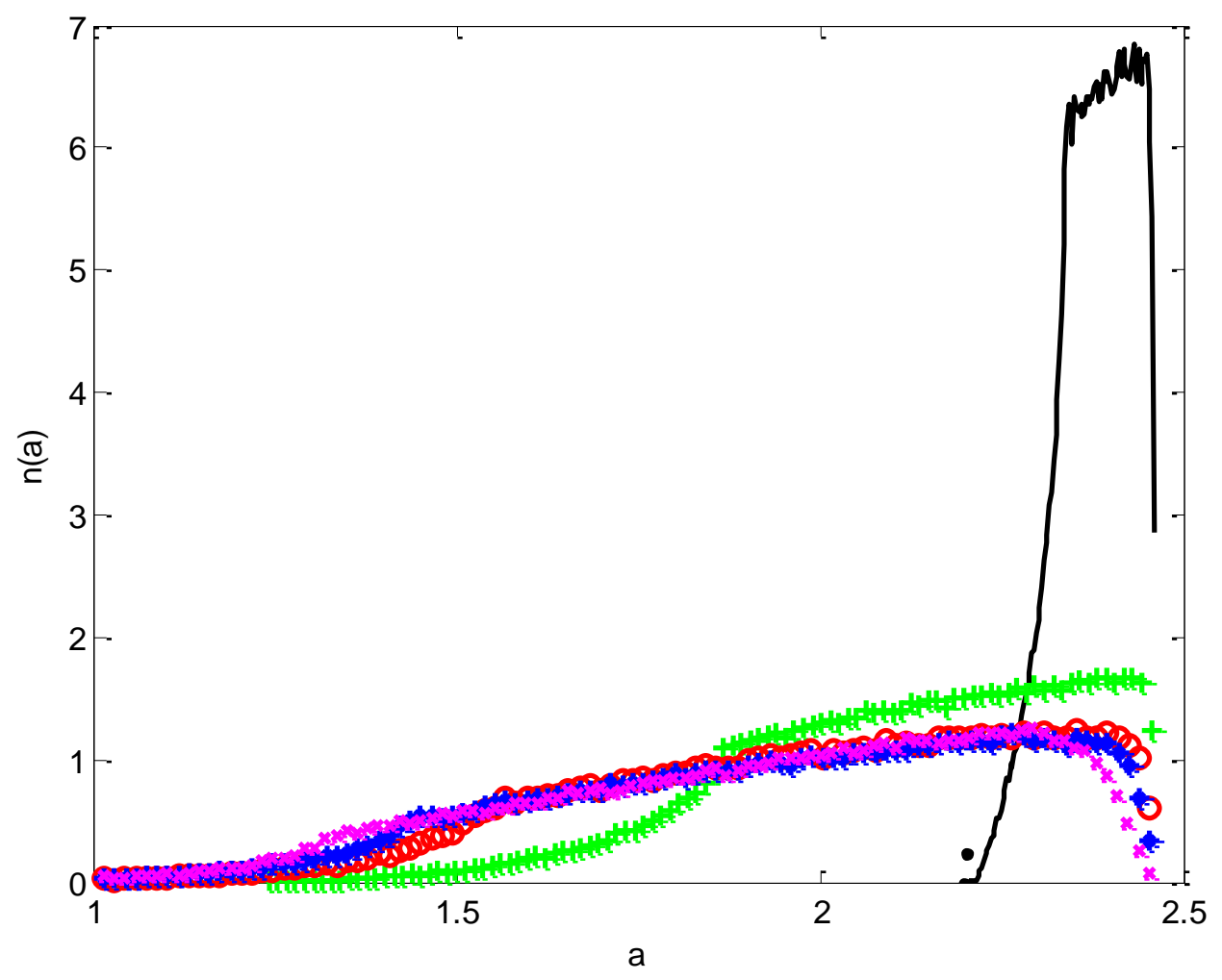

Figure 7a: pdf for $a$ 




Figure 7b: pdf for $b / a$ 


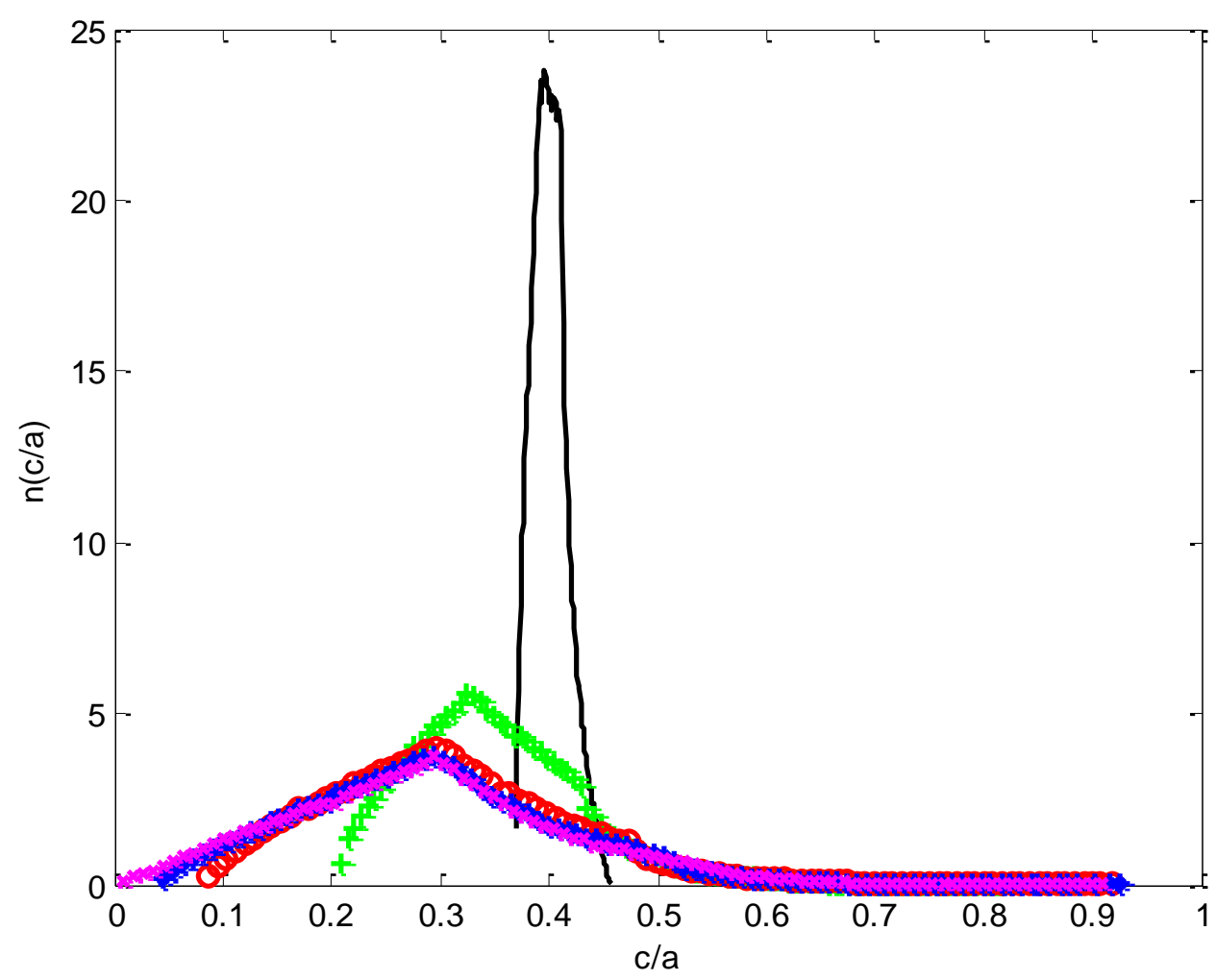

Figure 7c: pdf for $c / a$ 


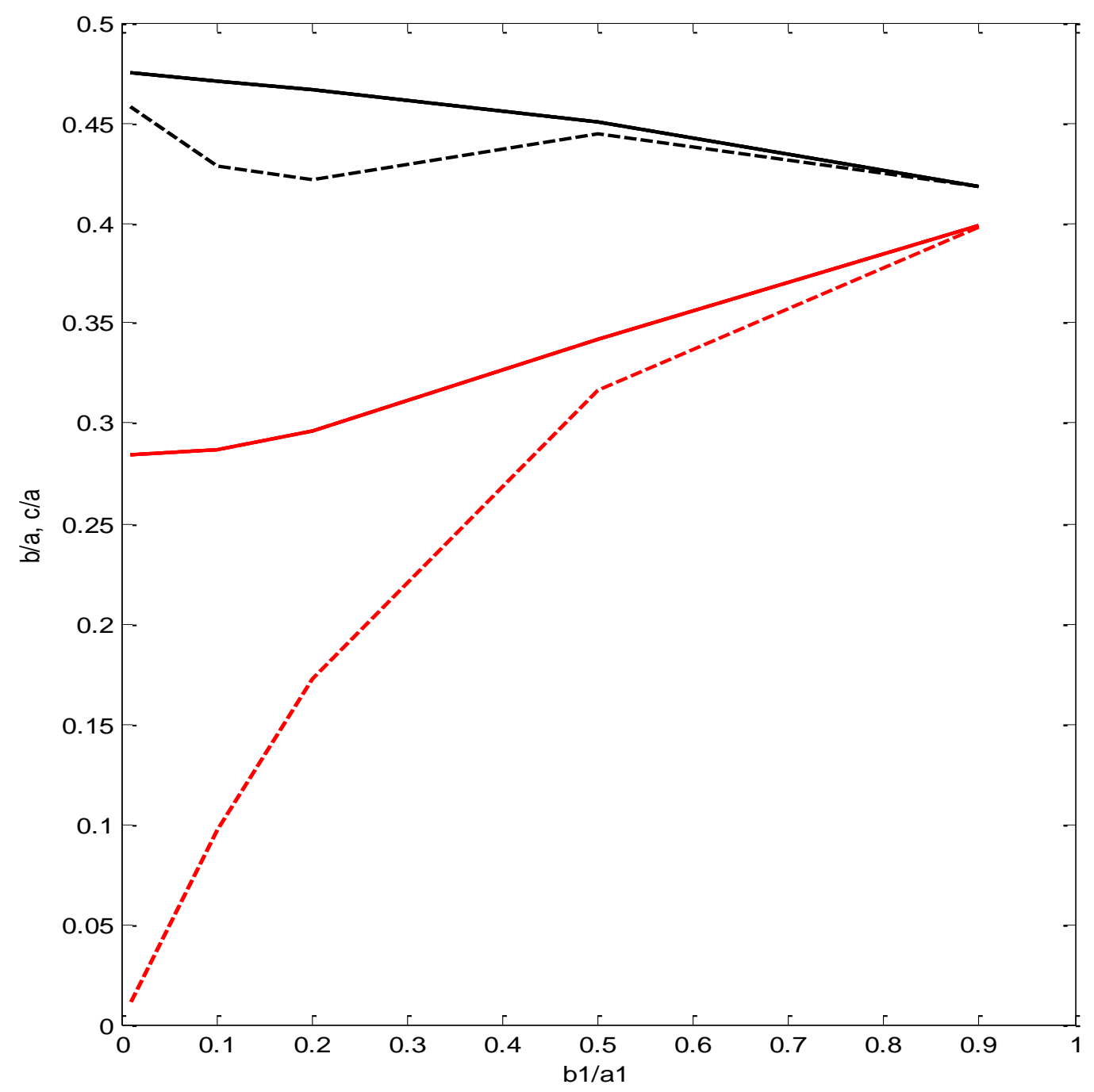

Figure 8: Collision of two identical prolate or oblate spheroids: elongation parameters of the average equivalent ellipsoid

Spheroids $\left(1,1, b_{1}, m_{l}=1\right)$ solid line; $\left(1, b_{1}, b_{1}, m_{l}=1\right)$ dashed line with $b_{1} \in S=\{0.01 ; 0.1 ; 0.2 ; 0.5 ; 0.9\}$

Average equivalent ellipsoids: black curve: $b / a$; red curve: $c / a$ 




Figure 9: $K_{12, E E}(+)$ and $K_{12, S}\left(\right.$ o) versus $K_{12, C C}$. Black marker corresponds to ordered clusters; red marker corresponds to random clusters. The solid line corresponds to $\mathrm{y}=1.27 \mathrm{x}$ 


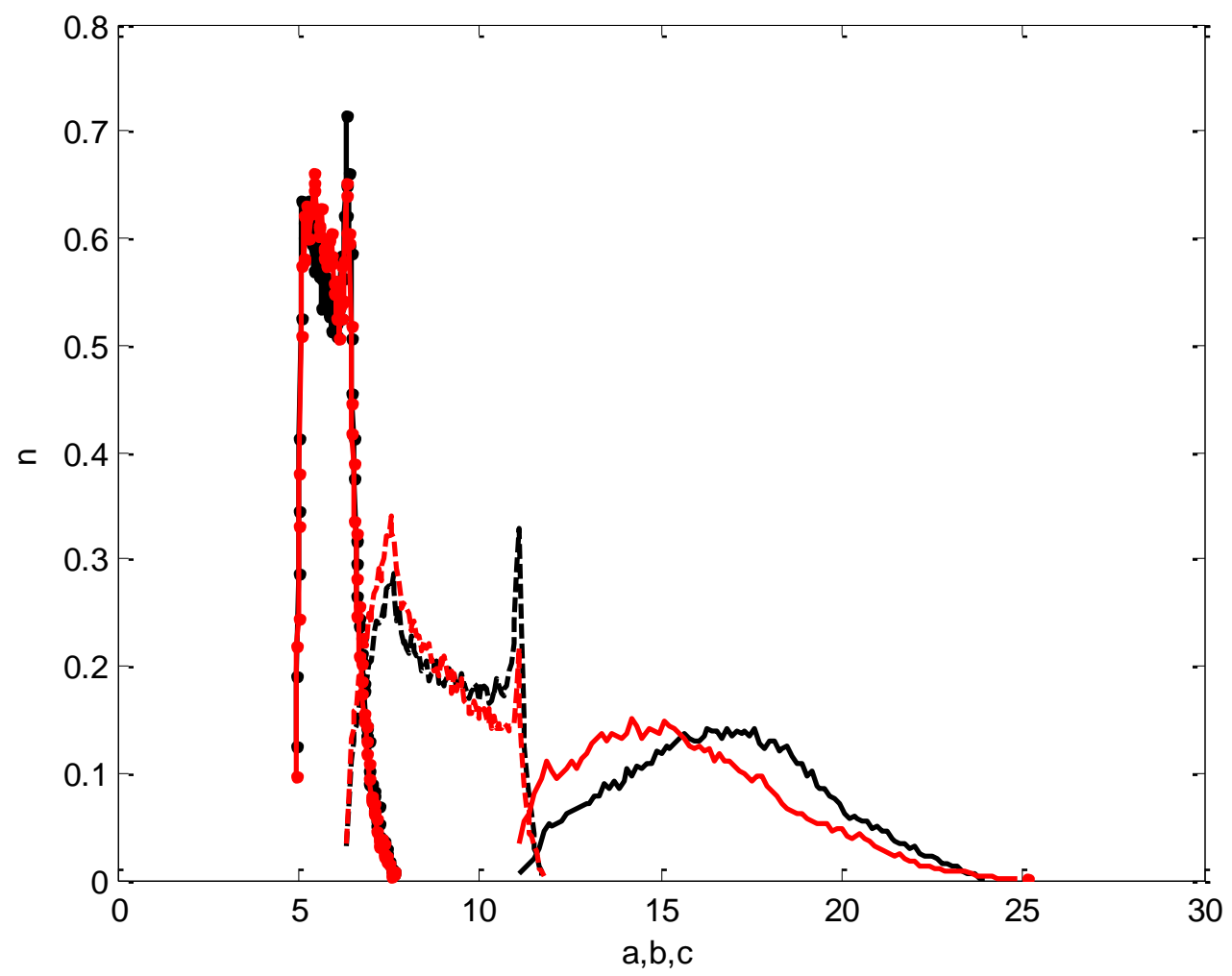

Figure 10a: pdf $n$ for $a, b, c$ parameters of equivalent ellipsoid for the collision of two random sphere clusters (A and B). $a$ : solid line; $b$ : dashed line; $c$ : dash-dot line.

Statistics from collisions between sphere clusters: red; Statistics from collisions between equivalent ellipsoids of clusters: black. 


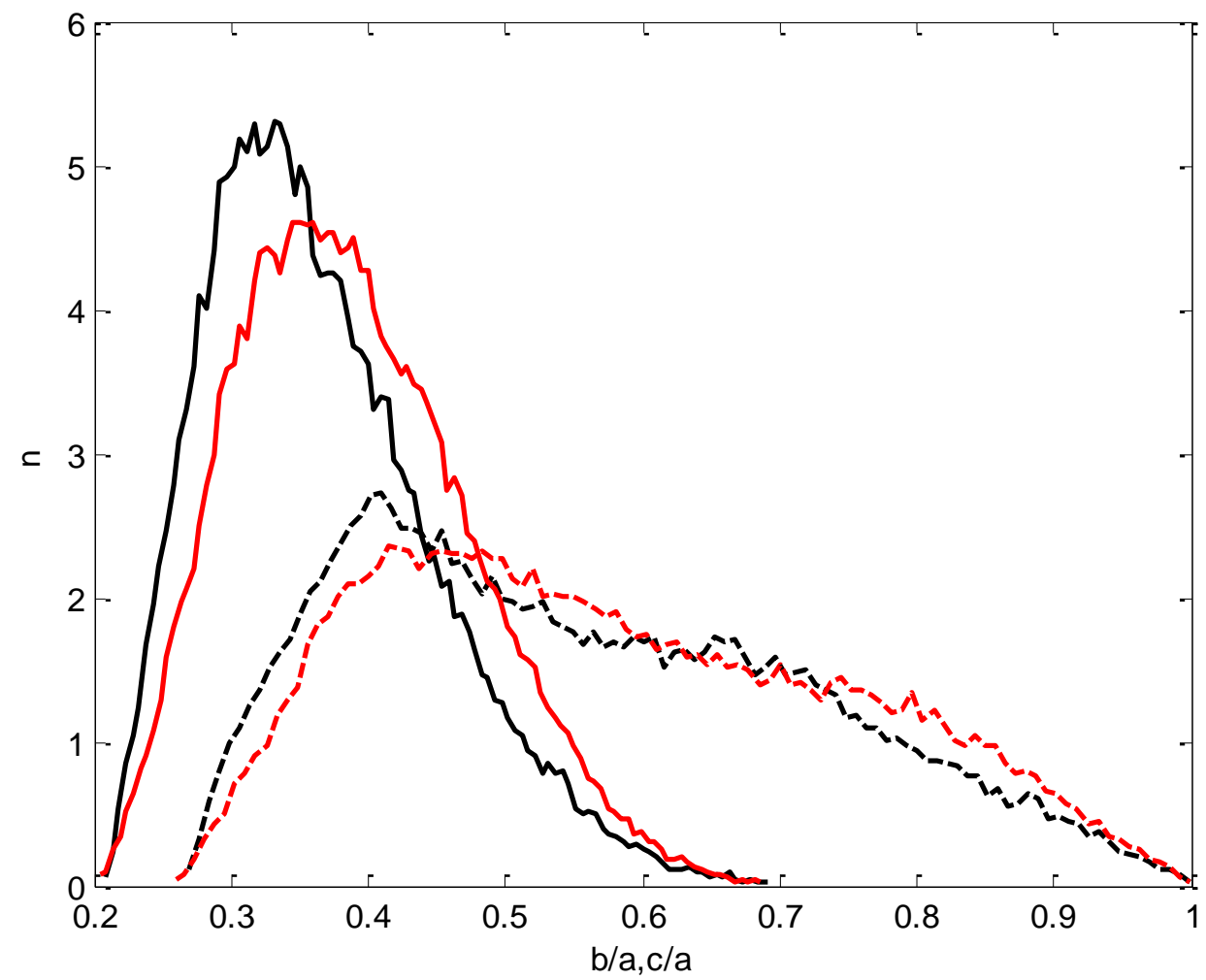

Figure 10b: pdf $n$ for $b / a$ and $c / a$ parameters of equivalent ellipsoid for the collision of two random sphere clusters (A and B). b/a: dashed line ; $c / a$ : solid line.

Statistics from collisions between sphere clusters: red; Statistics from collisions between equivalent ellipsoids of clusters. 


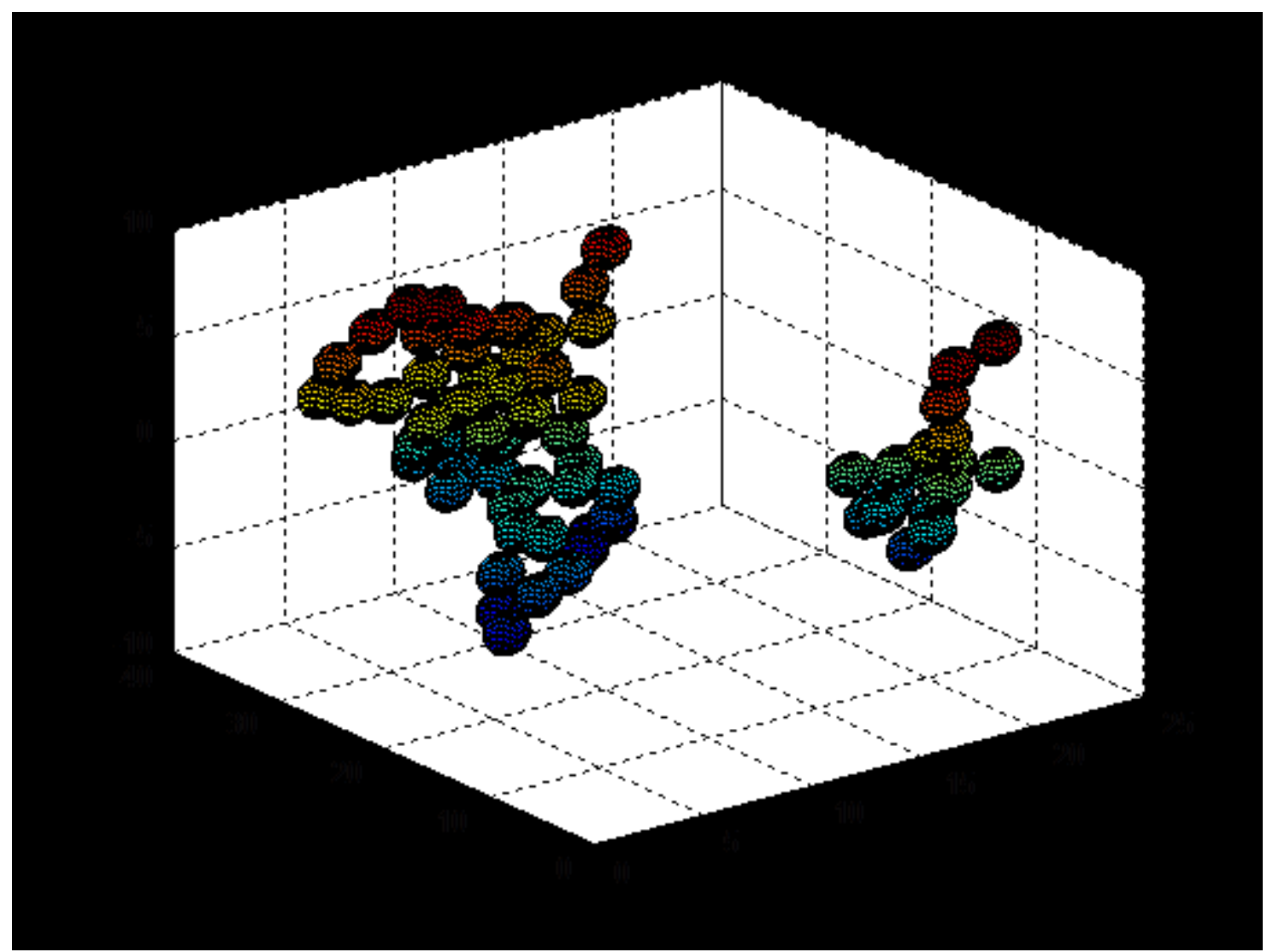

Figure 10c: Sphere cluster A (N=52) left sphere cluster $B(N=17)$ right 


\begin{tabular}{|l|l|l|l|l|l|l|l|l|l|}
\hline & $C_{1}$ & $C_{1,5}$ & $C_{1,6}$ & $C_{2,4}$ & $C_{2,5}$ & $C_{2,6}$ & $C_{3,4}$ & $C_{3,5}$ & $C_{3,6}$ \\
\hline$f_{b c}$ & -0.006 & 0.432 & 0.616 & 0.018 & -0.166 & -0.232 & 0.026 & 0.0014 & -0.814 \\
\hline$f_{a c}$ & 0.458 & 0.467 & 0.232 & 0.584 & -0.063 & 0.078 & 0.227 & -0.131 & 0.89 \\
\hline$f_{a b}$ & 0.457 & 0.1864 & 0.4724 & 0.2575 & -0.087 & 0.1807 & 0.4448 & -0.021 & 0.4845 \\
\hline
\end{tabular}

Table 1: optimized parameters for Eq.36 